Gazi University
Journal of Science
http://dergipark.gov.tr/gujs

\title{
Combination of Bipolar Soft Set and Soft Expert Set with Application in Decision Making
}

\author{
Orhan DALKILIC* (D), Naime DEMIRTAS \\ Mersin University, Faculty of Science, Department of Mathematics, 33433 Yenişsehir, Mersin
}

\author{
Highlights \\ - A mathematical model has been proposed by linking the soft expert set with the bipolarity logic. \\ - Basic set operations have been studied on the bipolar soft expert set. \\ - A new algorithm has been proposed to deal with the problems involving uncertainty.
}

\begin{tabular}{l} 
Article Info \\
\hline \\
Received: 19 Nov 2020 \\
Accepted: 30 June 2021 \\
Keywords \\
\hline Soft set \\
Soft expert set \\
Bipolar soft set \\
Bipolar soft expert set \\
Decision making
\end{tabular}

\section{INTRODUCTION}

One of the most important properties that must be addressed in order to perform data analysis in the most accurate way is uncertainty. However, the separations made in order to express the uncertainty correctly and thus to obtain the most ideal results are generally not so straightforward in this sense. Many mathematical models put forward to overcome this problem have been insufficient to be successful. There are many set types that have been brought to the literature in order to analyze the data in a near-ideal way. To give an example; the fuzzy set (briefly FS) [1], one of the pioneers of these set types, was proposed by Zadeh. In the following years, the rough set (briefly RS) [2] and intuitionistic fuzzy sets (briefly IFS) [3] can be expressed as remarkable theories in terms of decomposing uncertainty. However, there are some shortcomings in all of these theories. Molodtsov [4], who thinks that the main reason for these inadequacies is due to the lack of a parameterization tool, suggested soft set (briefly SS) theory. In addition to these, Molodtsov successfully applied it in many fields such as game theory, Riemann integration, smoothness of functions, theory of measurement and so on. The application area and diversity of the SS theory are rapidly increasing due to its success in expressing uncertainty [5-14].

Many versions of soft sets have been developed. One of these versions is the soft expert set (briefly SES) introduced by Alkhazaleh and Salleh [15]. This set type suggests that an expert group can be useful in the decision-making process. In this way, it is thought that more near-ideal results can be achieved in solving problems related to uncertainty. They also studied fuzzy SESs [16] by using SESs and fuzzy SSs. Then Enginoğlu and Dönmez [17] made some modifications to the SESs. Especially in recent years, interest in SS theory has been increased greatly, and many interesting applications of this theory have been expanded by embedding the ideas of mathematical models such as FS, IFS, interval-valued FS, N-SS, interval-valued fuzzy parameterized intuitionistic fuzzy SS [18-26]. 
Another mathematical model introduced to the literature as a result of the effort to express uncertain situations in an ideal way is the bipolar soft set (briefly BSS) theory proposed by Sabir and Naz [27]. BSS is an extended model of SS. It is a mathematical model that has great advantages in dealing with uncertain information and is proposed by including the idea of bipolarity in the SSs. Due to these advantages, many researches have been done on BSS theory, which has managed to attract the attention of many kinds of research [28-31].

In this paper, we examined the extension of BSSs and SESs and introduced the concept of bipolar soft expert set (briefly BSES). In other words, BSES theory, a new mathematical model, has been developed by examining the concept of bipolarity of information in the SES. This theory, it is aimed to obtain better results for uncertainty by providing more data from the decision-maker. We also discussed the operations of the BSES such as complement, subset, equal, AND, OR, restricted union, restricted intersection, extended union and extended intersection. Finally, a decision-making algorithm based on SSs has been proposed and an application has been given that illustrates how uncertainty situations can be expressed in an uncertainty problem by using this algorithm.

\section{PRELIMINARIES}

In this section, we recall some basic concepts in SS, SES and BSS. Detailed explanations related to SS, SES and BSS can be found in $[4,5,15,17,27]$.

Throughout this study, let $\mathrm{U}$ be an universe of objects and $2^{\mathrm{U}}$ denotes the power set of $\mathrm{U}$. Also, let $\mathrm{P}$ be a set of parameters and K, L, M be non-empty subsets of P.

Definition 2.1. [4] A pair $(\Gamma, \mathrm{K})$ is called an SS over $U$, where $\Gamma: \mathrm{K} \rightarrow 2^{\mathrm{U}}$ is a set valued mapping.

Definition 2.2. [5] Let $P=\left\{\mathrm{p}_{1}, \mathrm{p}_{2}, \ldots, \mathrm{p}_{\mathrm{n}}\right\}$ be a set of parameters. The NOT set of $\mathrm{P}$ denoted by $\neg \mathrm{P}$ is defined by $\neg \mathrm{P}=\left\{\neg \mathrm{p}_{1}, \neg \mathrm{p}_{2}, \ldots, \neg \mathrm{p}_{\mathrm{n}}\right\}$ where, $\neg \mathrm{p}_{\mathrm{i}}=$ not $\mathrm{p}_{\mathrm{i}}$ for all $\mathrm{i}$.

Now we present some basic notations for SESs. Let $\mathrm{E}$ be a set of experts and $\mathrm{O}$ be a set of opinions, $\mathrm{Z}=$ $\mathrm{P} \times \mathrm{E} \times \mathrm{O}$ and $\mathrm{K} \subseteq \mathrm{Z}$.

Definition 2.3. [15] A pair $(\Gamma, \mathrm{K})$ is called an SES over $\mathrm{U}$, where $\Gamma$ is a mapping given by $\Gamma: \mathrm{K} \rightarrow 2^{\mathrm{U}}$.

Definition 2.4. [15] For two SESs $(\Gamma, \mathrm{K})$ and $(\Lambda, \mathrm{L})$ over $U,(\Gamma, \mathrm{K})$ is called a soft expert subset of $(\Lambda, \mathrm{L})$ if $\mathrm{K} \subseteq \mathrm{L}$ and $\forall \mathrm{p} \in \mathrm{L}, \Lambda(\mathrm{p}) \subseteq \Gamma(\mathrm{p})$. This relationship is denoted by $(\Gamma, \mathrm{K}) \widetilde{\subseteq}(\Lambda, \mathrm{L})$.

Definition 2.5. [15] Two SESs $(\Gamma, \mathrm{K})$ and $(\Lambda, \mathrm{L})$ over $\mathrm{U}$ are said to be equal if $(\Gamma, \mathrm{K}) \widetilde{\subseteq}(\Lambda, \mathrm{L})$ and $(\Lambda, \mathrm{L}) \widetilde{\subseteq}(\Gamma, \mathrm{K})$.

Definition 2.6. [17] Let $\alpha=(\mathrm{p}, \mathrm{e}, \mathrm{o}) \in \mathrm{Z}$. Then not $\alpha$ and NOT Z are defined by $\neg \alpha=(\mathrm{p}, \mathrm{e}, 1-\mathrm{o})$ and $\neg \mathrm{Z}=\{\neg \alpha: \alpha \in \mathrm{Z}\}$, respectively. It can easily be seen that $\neg \mathrm{Z}=\mathrm{Z}$ but usually $\neg \mathrm{K} \neq \mathrm{K}$, for some $\mathrm{K} \subseteq \mathrm{Z}$.

Definition 2.7. [17] The complement of an SES $(\Gamma, K)$, denoted by $(\Gamma, K)^{\mathrm{c}}=\left(\Gamma^{\mathrm{c}}, \neg \mathrm{K}\right)$, is defined by $(\Gamma, \mathrm{K})^{\mathrm{c}}=\left(\Gamma^{\mathrm{c}}, \neg \mathrm{K}\right)$ where $\Gamma^{\mathrm{c}}: \neg \mathrm{K} \rightarrow 2^{\mathrm{U}}$ is a mapping given by $\Gamma^{\mathrm{C}}(\neg \alpha)=\mathrm{U}-\Gamma(\alpha)$, for all $\neg \alpha \in \neg \mathrm{K}$.

Definition 2.8. [15] Let $(\Gamma, K)$ be an SES over U. Then,

(1) An agree-SES $(\Gamma, \mathrm{K})_{1}$ over $\mathrm{U}$ is a soft expert subset of $(\Gamma, \mathrm{K})$ defined as follows:

$(\Gamma, \mathrm{K})_{1}=\left\{\Gamma_{1}(\alpha): \alpha \in \mathrm{P} \times \mathrm{E} \times\{1\}\right\}$

(2) A disagree-SES $(\Gamma, \mathrm{K})_{0}$ over $\mathrm{U}$ is a soft expert subset of $(\Gamma, \mathrm{K})$ defined as follows: 
$(\Gamma, \mathrm{K})_{0}=\left\{\Gamma_{0}(\alpha): \alpha \in \mathrm{P} \times \mathrm{E} \times\{0\}\right\}$

Definition 2.9. [15] If $(\Gamma, K)$ and $(\Lambda, L)$ are two SESs over $U$ then $(\Gamma, K)$ AND $(\Lambda, L)$ denoted by $(\Gamma, K) \wedge$ $(\Lambda, \mathrm{L})$, is defined by

$(\Gamma, \mathrm{K}) \wedge(\Lambda, \mathrm{L})=(\Omega, \mathrm{K} \times \mathrm{L})$

where $\Omega\left(\mathrm{p}^{\mathrm{k}}, \mathrm{p}^{\mathrm{l}}\right)=\Gamma\left(\mathrm{p}^{\mathrm{k}}\right) \cap \Lambda\left(\mathrm{p}^{\mathrm{l}}\right), \forall\left(\mathrm{p}^{\mathrm{k}}, \mathrm{p}^{\mathrm{l}}\right) \in \mathrm{K} \times \mathrm{L}$.

Definition 2.10. [15] If $(\Gamma, K)$ and $(\Lambda, L)$ are two SESs over $U$ then $(\Gamma, K)$ OR $(\Lambda, L)$ denoted by $(\Gamma, K) \vee$ $(\Lambda, L)$, is defined by

$(\Gamma, \mathrm{K}) \vee(\Lambda, \mathrm{L})=(\Omega, \mathrm{K} \times \mathrm{L})$

where $\Omega\left(\mathrm{p}^{\mathrm{k}}, \mathrm{p}^{\mathrm{l}}\right)=\Gamma\left(\mathrm{p}^{\mathrm{k}}\right) \cup \Lambda\left(\mathrm{p}^{\mathrm{l}}\right), \forall\left(\mathrm{p}^{\mathrm{k}}, \mathrm{p}^{\mathrm{l}}\right) \in \mathrm{K} \times \mathrm{L}$.

Definition 2.11. [15] The union of two SESs $(\Gamma, K)$ and $(\Lambda, L)$ over $U$ denoted by $(\Gamma, K) \widetilde{U}(\Lambda, L)$, is the $\operatorname{SES}(\Omega, M)$ where $\mathrm{M}=\mathrm{K} \cup \mathrm{L}, \forall \mathrm{p} \in \mathrm{M}$,

$\Omega(p)=\left\{\begin{array}{lc}\Gamma(p) & \text { if e } \in K-L \\ \Lambda(p) & \text { if } e \in L-K \\ \Gamma(p) \cup \Lambda(p) & \text { if } e \in K \cap L\end{array}\right.$

Definition 2.12. [15] The intersection of two SESs $(\Gamma, K)$ and $(\Lambda, L)$ over $U$ denoted by $(\Gamma, K) \widetilde{\cap}(\Lambda, L)$, is the $\operatorname{SES}(\Omega, M)$ where $M=K \cup L, \forall p \in M$,

$\Omega(p)=\left\{\begin{array}{lc}\Gamma(p) & \text { if e } \in K-L \\ \Lambda(p) & \text { if e } \in L-K \\ \Gamma(p) \cap \Lambda(p) & \text { if } e \in K \cap L\end{array}\right.$

Definition 2.13. [27] A triplet $(\Gamma, \Lambda, K)$ is called a BSS over $U$, where $\Gamma$ and $\Lambda$ are mappings, given by $\Gamma: \mathrm{K} \rightarrow 2^{\mathrm{U}}$ and $\Lambda: \neg \mathrm{L} \rightarrow 2^{\mathrm{U}}$ such that $\Gamma(\mathrm{p}) \cap \Lambda(\neg \mathrm{p})=\varnothing, \forall \mathrm{p} \in \mathrm{K}$.

Definition 2.14. [27] For two BSSs $(\Gamma, \Lambda, K)$ and $\left(\Gamma_{1}, \Lambda_{1}, \mathrm{~L}\right)$ over $U$, we say that $(\Gamma, \Lambda, K)$ is a bipolar soft subset of $\left(\Gamma_{1}, \Lambda_{1}, L\right)$ if,

(1) $\mathrm{K} \subseteq \mathrm{L}$ and

(2) $\Gamma(\mathrm{p}) \subseteq \Gamma_{1}(\mathrm{p})$ and $\Lambda_{1}(\neg \mathrm{p}) \subseteq \Lambda(\neg \mathrm{p}), \forall \mathrm{p} \in \mathrm{K}$.

This relationship is denoted by $(\Gamma, \Lambda, \mathrm{K}) \widetilde{\widetilde{ }}\left(\Gamma_{1}, \Lambda_{1}, \mathrm{~L}\right)$. They are said to be equal if $(\Gamma, \Lambda, \mathrm{K}) \widetilde{\subseteq}\left(\Gamma_{1}, \Lambda_{1}, \mathrm{~L}\right)$

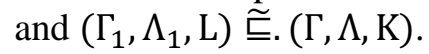

Definition 2.15. [27] The complement of a BSS $(\Gamma, \Lambda, K)$, denoted by $(\Gamma, \Lambda, K)^{\tilde{c}}$, is defined by $(\Gamma, \Lambda, K)^{\tilde{c}}=$ $\left(\Gamma^{\tilde{\mathrm{c}}}, \Lambda^{\tilde{\mathrm{c}}}, \mathrm{K}\right)$ where $\Gamma^{\tilde{\mathrm{c}}}$ and $\Lambda^{\tilde{\mathrm{c}}}$ are mappings given by $\Gamma^{\tilde{\mathrm{c}}}(\mathrm{p})=\Lambda(\neg \mathrm{p})$ and $\Lambda^{\tilde{\mathrm{c}}}(\neg \mathrm{p})=\Gamma(\mathrm{p}), \forall \mathrm{p} \in \mathrm{K}$.

Definition 2.16. [27] If $(\Gamma, \Lambda, K)$ and $\left(\Gamma_{1}, \Lambda_{1}, L\right)$ are two BSSs over $U$ then " $(\Gamma, \Lambda, K)$ AND $\left(\Gamma_{1}, \Lambda_{1}, L\right)$ " denoted $(\Gamma, \Lambda, K) \widetilde{\Lambda}\left(\Gamma_{1}, \Lambda_{1}, \mathrm{~L}\right)$ is defined by

$(\Gamma, \Lambda, \mathrm{K}) \tilde{\Lambda}\left(\Gamma_{1}, \Lambda_{1}, \mathrm{~L}\right)=\left(\Gamma_{2}, \Lambda_{2}, \mathrm{~K} \times \mathrm{L}\right)$

where $\Gamma_{2}\left(\mathrm{p}^{\mathrm{k}}, \mathrm{p}^{\mathrm{l}}\right)=\Gamma\left(\mathrm{p}^{\mathrm{k}}\right) \cap \Gamma_{1}\left(\mathrm{p}^{\mathrm{l}}\right)$ and $\Lambda_{2}\left(\neg \mathrm{p}^{\mathrm{k}}, \neg \mathrm{p}^{\mathrm{l}}\right)=\Lambda\left(\neg \mathrm{p}^{\mathrm{k}}\right) \cup \Lambda_{1}\left(\neg \mathrm{p}^{\mathrm{l}}\right), \forall\left(\mathrm{p}^{\mathrm{k}}, \mathrm{p}^{\mathrm{l}}\right) \in \mathrm{K} \times \mathrm{L}$. 
Definition 2.17. [27] If $(\Gamma, \Lambda, K)$ and $\left(\Gamma_{1}, \Lambda_{1}, \mathrm{~L}\right)$ are two BSSs over $\mathrm{U}$ then " $(\Gamma, \Lambda, \mathrm{K}) \mathrm{OR}\left(\Gamma_{1}, \Lambda_{1}, \mathrm{~L}\right) "$ denoted $(\Gamma, \Lambda, K) \widetilde{V}\left(\Gamma_{1}, \Lambda_{1}, \mathrm{~L}\right)$ is defined by

$(\Gamma, \Lambda, \mathrm{K}) \widetilde{\mathrm{V}}\left(\Gamma_{1}, \Lambda_{1}, \mathrm{~L}\right)=\left(\Gamma_{2}, \Lambda_{2}, \mathrm{~K} \times \mathrm{L}\right)$

where $\Gamma_{2}\left(\mathrm{p}^{\mathrm{k}}, \mathrm{p}^{\mathrm{l}}\right)=\Gamma\left(\mathrm{p}^{\mathrm{k}}\right) \cup \Gamma_{1}\left(\mathrm{p}^{\mathrm{l}}\right)$ and $\Lambda_{2}\left(\neg \mathrm{p}^{\mathrm{k}}, \neg \mathrm{p}^{\mathrm{l}}\right)=\Lambda\left(\neg \mathrm{p}^{\mathrm{k}}\right) \cap \Lambda_{1}\left(\neg \mathrm{p}^{\mathrm{l}}\right), \forall\left(\mathrm{p}^{\mathrm{k}}, \mathrm{p}^{\mathrm{l}}\right) \in \mathrm{K} \times \mathrm{L}$.

Definition 2.18. [27] Let $(\Gamma, \Lambda, K)$ and $\left(\Gamma_{1}, \Lambda_{1}, L\right)$ be BSSs over $U$. Then,

(1) The extended union of $(\Gamma, \Lambda, K)$ and $\left(\Gamma_{1}, \Lambda_{1}, \mathrm{~L}\right)$, denoted by $(\Gamma, \Lambda, \mathrm{K}) \widetilde{\mathrm{U}}\left(\Gamma_{1}, \Lambda_{1}, \mathrm{~L}\right)$, is defined as the BSS $\left(\Gamma_{2}, \Lambda_{2}, M\right)$ over $U$, where $M=K \cup L$ and $\forall p \in M$,

$\Gamma_{2}(e)=\left\{\begin{array}{lc}\Gamma(p) & \text { if } p \in K-L \\ \Gamma_{1}(p) & \text { if } p \in L-K \\ \Gamma(e) \cup \Gamma_{1}(e) & \text { if } p \in K \cap L\end{array}\right.$

$\Lambda_{2}(\neg \mathrm{e})= \begin{cases}\Lambda(\neg \mathrm{p}) & \text { if } \neg \mathrm{p} \in \mathrm{K}-\mathrm{L} \\ \Lambda_{1}(\neg \mathrm{p}) & \text { if } \neg \mathrm{p} \in \mathrm{L}-\mathrm{K} \\ \Lambda(\neg \mathrm{p}) \cap \Lambda_{1}(\neg \mathrm{p}) & \text { if } \neg \mathrm{p} \in \mathrm{K} \cap \mathrm{L}\end{cases}$

(2) The extended intersection of $(\Gamma, \Lambda, K)$ and $\left(\Gamma_{1}, \Lambda_{1}, L\right)$, denoted by $(\Gamma, \Lambda, K) \widetilde{\Gamma}\left(\Gamma_{1}, \Lambda_{1}, L\right)$, is defined as the BSS $\left(\Gamma_{2}, \Lambda_{2}, M\right)$ over $\mathrm{U}$, where $\mathrm{M}=\mathrm{K} \cup \mathrm{L}$ and $\forall \mathrm{p} \in \mathrm{M}$,

$\Gamma_{2}(\mathrm{e})=\left\{\begin{array}{lc}\Gamma(\mathrm{p}) & \text { if } \mathrm{p} \in \mathrm{K}-\mathrm{L} \\ \Gamma_{1}(\mathrm{p}) & \text { if } \mathrm{p} \in \mathrm{L}-\mathrm{K}, \\ \Gamma(\mathrm{e}) \cap \Gamma_{1}(\mathrm{e}) & \text { if } \mathrm{p} \in \mathrm{K} \cap \mathrm{L}\end{array}\right.$

$\Lambda_{2}(\neg \mathrm{e})= \begin{cases}\Lambda(\neg \mathrm{p}) & \text { if } \neg \mathrm{p} \in \mathrm{K}-\mathrm{L} \\ \Lambda_{1}(\neg \mathrm{p}) & \text { if } \neg \mathrm{p} \in \mathrm{L}-\mathrm{K} \\ \Lambda(\neg \mathrm{p}) \cup \Lambda_{1}(\neg \mathrm{p}) & \text { if } \neg \mathrm{p} \in \mathrm{K} \cap \mathrm{L}\end{cases}$

(3) The restricted union of $(\Gamma, \Lambda, K)$ and $\left(\Gamma_{1}, \Lambda_{1}, \mathrm{~L}\right)$, denoted by $(\Gamma, \Lambda, K) \sqcup_{\Re}\left(\Gamma_{1}, \Lambda_{1}, \mathrm{~L}\right)$, is defined as the BSS $\left(\Gamma_{2}, \Lambda_{2}, M\right)$ over $U$, where $M=K \cap L$ is non-empty and $\forall p \in M$,

$\Gamma_{2}(\mathrm{p})=\Gamma(\mathrm{p}) \cup \Lambda(\mathrm{p})$,

$\Lambda_{2}(\neg \mathrm{p})=\Gamma_{1}(\neg \mathrm{p}) \cap \Lambda_{1}(\neg \mathrm{p})$.

(4) The restricted intersection of $(\Gamma, \Lambda, K)$ and $\left(\Gamma_{1}, \Lambda_{1}, L\right)$, denoted by $(\Gamma, \Lambda, K) \Pi_{\Re}\left(\Gamma_{1}, \Lambda_{1}, L\right)$, is defined as the BSS $\left(\Gamma_{2}, \Lambda_{2}, M\right)$ over $U$, where $M=K \cap L$ is non-empty and $\forall p \in M$,

$\Gamma_{2}(p)=\Gamma(p) \cap \Lambda(p)$,

$\Lambda_{2}(\neg \mathrm{p})=\Gamma_{1}(\neg \mathrm{p}) \cup \Lambda_{1}(\neg \mathrm{p})$.

\section{BIPOLAR SOFT EXPERT SETS}

In this section, we introduce a new mathematical model, bipolar soft expert set (briefly BSES), to express uncertainty problems in a more ideal way and give some basic operations such as complement, subset, equal, AND, OR, extended union, extended intersection, restricted union and restricted intersection. Then, some basic properties of these concepts are given. 
Let $\mathrm{E}$ be a set of experts, $\mathrm{O}=\{0,1\}$ be a set of opinions, $\mathrm{Z}=\mathrm{P} \times \mathrm{E} \times \mathrm{O}$ and $\mathrm{K}, \mathrm{L}, \mathrm{M} \subseteq \mathrm{Z}$.

Remark 3.1. For simplicity, in this paper we assume that there are two-valued opinions only in set 0 , that is, $O=\{0,1\}=$ disagree, agree $\}$, but multivalued opinions may be assumed as well.

Definition 3.1. A triplet $(\Gamma, \Lambda, \mathrm{K})$ is called a BSES over $\mathrm{U}$, where $\Gamma$ and $\Lambda$ are mappings, given by $\Gamma: \mathrm{K} \rightarrow$ $2^{\mathrm{U}}$ and $\Lambda: \neg \mathrm{K} \rightarrow 2^{\mathrm{U}}$ such that $\Gamma(\mathrm{p}, \mathrm{e}, 1) \cap \Lambda(\neg \mathrm{p}, \mathrm{e}, 1)=\emptyset$ or $\Gamma(\mathrm{p}, \mathrm{e}, 0) \cap \Lambda(\neg \mathrm{p}, \mathrm{e}, 0)=\emptyset$ for all $(\mathrm{p}, \mathrm{e}, \mathrm{o}) \in \mathrm{K}$ and $(\neg \mathrm{p}, \mathrm{e}, \mathrm{o}) \in \neg \mathrm{K}$. Here;

$\Gamma(\mathrm{p}, \mathrm{e}, 1)$ : the set of objects that provide the parameter $p$ by expert $e$, $\Lambda(\neg \mathrm{p}, \mathrm{e}, 1)$ : the set of objects that provide the parameter $\neg \mathrm{p}$ by expert $e$, $\Gamma(\mathrm{p}, \mathrm{e}, 0)$ : the set of objects that do not provide the parameter $p$ by expert $e$, $\Lambda(\neg \mathrm{p}, \mathrm{e}, 0)$ : the set of objects that do not provide the parameter $\neg$ p by expert $e$.

Here $(\Gamma, \mathrm{K})$ and $(\Lambda, \neg \mathrm{K})$ are SESs, since $\mathrm{K} \subseteq \mathrm{Z}=\mathrm{P} \times \mathrm{E} \times 0$.

Example 3.1. Since people's needs and desires in general are different, it is a difficult task for a person to choose the right car. When choosing a car, the impact of many factors affects their decision-making. For this, a private company wants to get help from two experts in this field to increase its profits. Let $U=$ $\left\{\mathrm{u}_{1}, \mathrm{u}_{2}, \mathrm{u}_{3}, \mathrm{u}_{4}, \mathrm{u}_{5}\right\}$ be the set of hybrid cars under consideration, $\mathrm{P}=\left\{\mathrm{p}_{1}, \mathrm{p}_{2}\right\}=\{$ durability, fuel efficient $\}$ and $\neg \mathrm{P}=\left\{\neg \mathrm{p}_{1}, \neg \mathrm{p}_{2}\right\}=\{$ non - durable, fuel inefficient $\}$ be the set of parameters and $\mathrm{E}=\left\{\mathrm{e}_{1}, \mathrm{e}_{2}\right\}$ be the set of experts the company has consulted. Suppose that the opinions expressed by the experts about the cars in the private company are as follows:

$$
\begin{aligned}
& \Gamma\left(\mathrm{p}_{1}, \mathrm{e}_{1}, 1\right)=\left\{\mathrm{u}_{2}, \mathrm{u}_{3}\right\}, \quad \Lambda\left(\neg \mathrm{p}_{1}, \mathrm{e}_{1}, 1\right)=\left\{\mathrm{u}_{4}\right\}, \quad \Gamma\left(\mathrm{p}_{1}, \mathrm{e}_{2}, 1\right)=\left\{\mathrm{u}_{3}, \mathrm{u}_{5}\right\}, \quad \Lambda\left(\neg \mathrm{p}_{1}, \mathrm{e}_{2}, 1\right)= \\
& \left\{\mathrm{u}_{1}, \mathrm{u}_{4}\right\}, \quad \Gamma\left(\mathrm{p}_{2}, \mathrm{e}_{1}, 1\right)=\left\{\mathrm{u}_{2}, \mathrm{u}_{3}, \mathrm{u}_{5}\right\}, \quad \Lambda\left(\neg \mathrm{p}_{2}, \mathrm{e}_{1}, 1\right)=\left\{\mathrm{u}_{1}, \mathrm{u}_{4}\right\}, \quad \Gamma\left(\mathrm{p}_{2}, \mathrm{e}_{2}, 1\right)= \\
& \left\{\mathrm{u}_{2}, \mathrm{u}_{5}\right\}, \quad \Lambda\left(\neg \mathrm{p}_{2}, \mathrm{e}_{2}, 1\right)=\left\{\mathrm{u}_{1}\right\}, \quad \Gamma\left(\mathrm{p}_{1}, \mathrm{e}_{1}, 0\right)=\left\{\mathrm{u}_{1}, \mathrm{u}_{4}, \mathrm{u}_{5}\right\}, \\
& \Lambda\left(\neg \mathrm{p}_{1}, \mathrm{e}_{1}, 0\right)=\left\{\mathrm{u}_{1}, \mathrm{u}_{2}, \mathrm{u}_{3}, \mathrm{u}_{5}\right\}, \Gamma\left(\mathrm{p}_{1}, \mathrm{e}_{2}, 0\right)=\left\{\mathrm{u}_{1}, \mathrm{u}_{4}, \mathrm{u}_{5}\right\}, \quad \Lambda\left(\neg \mathrm{p}_{1}, \mathrm{e}_{2}, 0\right)=\left\{\mathrm{u}_{2}, \mathrm{u}_{3}, \mathrm{u}_{5}\right\}, \\
& \Gamma\left(\mathrm{p}_{2}, \mathrm{e}_{1}, 0\right)=\left\{\mathrm{u}_{1}, \mathrm{u}_{4}\right\}, \quad \Lambda\left(\neg \mathrm{p}_{2}, \mathrm{e}_{1}, 0\right)=\left\{\mathrm{u}_{2}, \mathrm{u}_{3}, \mathrm{u}_{5}\right\}, \Gamma\left(\mathrm{p}_{2}, \mathrm{e}_{2}, 0\right)=\left\{\mathrm{u}_{1}, \mathrm{u}_{3}, \mathrm{u}_{4}\right\}, \\
& \Lambda\left(\neg \mathrm{p}_{2}, \mathrm{e}_{2}, 0\right)=\left\{\mathrm{u}_{2}, \mathrm{u}_{3}, \mathrm{u}_{4}, \mathrm{u}_{5}\right\} .
\end{aligned}
$$

All these opinions expressed by experts can be expressed with the help of the BSES $(\Gamma, \Lambda, Z)$ as follows:

$$
(\Gamma, \Lambda, Z)=\left\{\begin{array}{c}
\left(\left(\mathrm{p}_{1}, \mathrm{e}_{1}, 1\right),\left\{\mathrm{u}_{2}, \mathrm{u}_{3}\right\}\right),\left(\left(\neg \mathrm{p}_{1}, \mathrm{e}_{1}, 1\right),\left\{\mathrm{u}_{4}\right\}\right),\left(\left(\mathrm{p}_{1}, \mathrm{e}_{2}, 1\right),\left\{\mathrm{u}_{3}, \mathrm{u}_{5}\right\}\right), \\
\left(\left(\neg \mathrm{p}_{1}, \mathrm{e}_{2}, 1\right),\left\{\mathrm{u}_{1}, \mathrm{u}_{4}\right\}\right),\left(\left(\mathrm{p}_{2}, \mathrm{e}_{2}, 1\right),\left\{\mathrm{u}_{2}, \mathrm{u}_{5}\right\}\right),\left(\left(\neg \mathrm{p}_{2}, \mathrm{e}_{2}, 1\right),\left\{\mathrm{u}_{1}\right\}\right), \\
\left(\left(\mathrm{p}_{1}, \mathrm{e}_{1}, 0\right),\left\{\mathrm{u}_{1}, \mathrm{u}_{4}, \mathrm{u}_{5}\right\}\right),\left(\left(\neg \mathrm{p}_{1}, \mathrm{e}_{2}, 0\right),\left\{\mathrm{u}_{2}, \mathrm{u}_{3}, \mathrm{u}_{5}\right\}\right),\left(\left(\mathrm{p}_{2}, \mathrm{e}_{1}, 0\right),\left\{\mathrm{u}_{1}, \mathrm{u}_{4}\right\}\right), \\
\left(\left(\neg \mathrm{p}_{2}, \mathrm{e}_{1}, 0\right),\left\{\mathrm{u}_{2}, \mathrm{u}_{3}, \mathrm{u}_{5}\right\}\right),\left(\left(\mathrm{p}_{2}, \mathrm{e}_{2}, 0\right),\left\{\mathrm{u}_{1}, \mathrm{u}_{3}, \mathrm{u}_{4}\right\}\right),\left(\left(\neg \mathrm{p}_{2}, \mathrm{e}_{2}, 0\right),\left\{\mathrm{u}_{2}, \mathrm{u}_{3}, \mathrm{u}_{4}, \mathrm{u}_{5}\right\}\right)
\end{array}\right\} .
$$

Here $(\Gamma, \Lambda, Z)$ is a BSES over $U$.

Definition 3.2. For two BSESs $(\Gamma, \Lambda, K)$ and $\left(\Gamma_{1}, \Lambda_{1}, \mathrm{~L}\right)$ over $\mathrm{U}$, we say that $(\Gamma, \Lambda, \mathrm{K})$ is a bipolar soft expert subset of $\left(\Gamma_{1}, \Lambda_{1}, L\right)$ if

(1) $\mathrm{K} \subseteq \mathrm{L}$ and

(2) $\Gamma(\mathrm{p}, \mathrm{e}, \mathrm{o}) \subseteq \Gamma_{1}(\mathrm{p}, \mathrm{e}, \mathrm{o})$ and $\Lambda_{1}(\neg \mathrm{p}, \mathrm{e}, \mathrm{o}) \subseteq \Lambda(\neg \mathrm{p}, \mathrm{e}, \mathrm{o})$ for all $(\mathrm{p}, \mathrm{e}, \mathrm{o}) \in \mathrm{K} \subseteq \mathrm{P} \times \mathrm{E} \times 0$.

This relationship is denoted by $(\Gamma, \Lambda, \mathrm{K}) \hat{\subseteq}\left(\Gamma_{1}, \Lambda_{1}, \mathrm{~L}\right)$.

Definition 3.3. [25] Two BSESs $(\Gamma, \Lambda, K)$ and $\left(\Gamma_{1}, \Lambda_{1}, \mathrm{~L}\right)$ over $\mathrm{U}$ are said to be equal if $(\Gamma, \Lambda, \mathrm{K}) \hat{\subseteq}\left(\Gamma_{1}, \Lambda_{1}, \mathrm{~L}\right)$ and $\left(\Gamma_{1}, \Lambda_{1}, \mathrm{~L}\right) \hat{\subseteq}(\Gamma, \Lambda, \mathrm{K})$.

Example 3.2. Consider Example 3.1 and suppose that the private company consults the same experts again after a certain period of time. Then, 
and

$$
\mathrm{K}=\left\{\left(\mathrm{p}_{1}, \mathrm{e}_{1}, 1\right),\left(\neg \mathrm{p}_{1}, \mathrm{e}_{1}, 1\right),\left(\mathrm{p}_{2}, \mathrm{e}_{1}, 0\right),\left(\neg \mathrm{p}_{2}, \mathrm{e}_{1}, 0\right),\left(\mathrm{p}_{2}, \mathrm{e}_{2}, 1\right),\left(\neg \mathrm{p}_{2}, \mathrm{e}_{2}, 1\right)\right\}
$$

$$
L=\left\{\left(p_{1}, e_{1}, 1\right),\left(\neg p_{1}, e_{1}, 1\right),\left(p_{2}, e_{2}, 1\right),\left(\neg p_{2}, e_{2}, 1\right)\right\}
$$

Clearly $\mathrm{L} \subseteq \mathrm{K}$. Let $(\Gamma, \Lambda, \mathrm{K})$ and $\left(\Gamma_{1}, \Lambda_{1}, \mathrm{~L}\right)$ be defined as follows:

$$
\begin{gathered}
(\Gamma, \Lambda, K)=\left\{\begin{array}{c}
\left(\left(\mathrm{p}_{1}, \mathrm{e}_{1}, 1\right),\left\{\mathrm{u}_{2}, \mathrm{u}_{3}\right\}\right),\left(\left(\neg \mathrm{p}_{1}, \mathrm{e}_{1}, 1\right),\left\{\mathrm{u}_{4}\right\}\right),\left(\left(\mathrm{p}_{2}, \mathrm{e}_{1}, 0\right),\left\{\mathrm{u}_{1}, \mathrm{u}_{4}\right\}\right), \\
\left(\left(\neg \mathrm{p}_{2}, \mathrm{e}_{1}, 0\right),\left\{\mathrm{u}_{2}, \mathrm{u}_{3}, \mathrm{u}_{5}\right\}\right),\left(\left(\mathrm{p}_{2}, \mathrm{e}_{2}, 1\right),\left\{\mathrm{u}_{2}, \mathrm{u}_{5}\right\}\right),\left(\left(\neg \mathrm{p}_{2}, \mathrm{e}_{2}, 1\right),\left\{\mathrm{u}_{1}\right\}\right)
\end{array}\right\}, \\
\left(\Gamma_{1}, \Lambda_{1}, \mathrm{~L}\right)=\left\{\left(\left(\mathrm{p}_{1}, \mathrm{e}_{1}, 1\right),\left\{\mathrm{u}_{2}, \mathrm{u}_{3}\right\}\right),\left(\left(\neg \mathrm{p}_{1}, \mathrm{e}_{1}, 1\right),\left\{\mathrm{u}_{4}\right\}\right),\left(\left(\mathrm{p}_{2}, \mathrm{e}_{2}, 1\right),\left\{\mathrm{u}_{2}, \mathrm{u}_{5}\right\}\right),\left(\left(\neg \mathrm{p}_{2}, \mathrm{e}_{2}, 1\right),\left\{\mathrm{u}_{1}\right\}\right)\right\} .
\end{gathered}
$$

Therefore $\left(\Gamma_{1}, \Lambda_{1}, \mathrm{~L}\right) \hat{\subseteq}(\Gamma, \Lambda, \mathrm{K})$.

Definition 3.4. An agree-BSES $(\Gamma, \Lambda, K)_{1}$ over $U$ is a bipolar soft expert subset of $(\Gamma, \Lambda, K)$ defined as follows:

$$
(\Gamma, \Lambda, \mathrm{K})_{1}=\{\Gamma(\mathrm{p}, \mathrm{e}, 1) \cup \Lambda(\neg \mathrm{p}, \mathrm{e}, 1): \quad \mathrm{p} \in \mathrm{P}, \neg \mathrm{p} \in \neg \mathrm{P}, \mathrm{e} \in \mathrm{E}\} .
$$

Definition 3.5. An disagree-BSES $(\Gamma, \Lambda, K)_{0}$ over $U$ is a bipolar soft expert subset of $(\Gamma, \Lambda, K)$ defined as follows:

$$
(\Gamma, \Lambda, \mathrm{K})_{0}=\{\Gamma(\mathrm{p}, \mathrm{e}, 0) \cup \Lambda(\neg \mathrm{p}, \mathrm{e}, 0): \quad \mathrm{p} \in \mathrm{P}, \neg \mathrm{p} \in \neg \mathrm{P}, \mathrm{e} \in \mathrm{E}\} .
$$

Example 3.3. Consider Example 3.1. Then the agree-BSES $(\Gamma, \Lambda, K)_{1}$ over $U$ is

$$
(\Gamma, \Lambda, K)_{1}=\left\{\begin{array}{c}
\left(\left(\mathrm{p}_{1}, \mathrm{e}_{1}, 1\right),\left\{\mathrm{u}_{2}, \mathrm{u}_{3}\right\}\right),\left(\left(\neg \mathrm{p}_{1}, \mathrm{e}_{1}, 1\right),\left\{\mathrm{u}_{4}\right\}\right),\left(\left(\mathrm{p}_{1}, \mathrm{e}_{2}, 1\right),\left\{\mathrm{u}_{3}, \mathrm{u}_{5}\right\}\right), \\
\left(\left(\neg \mathrm{p}_{1}, \mathrm{e}_{2}, 1\right),\left\{\mathrm{u}_{1}, \mathrm{u}_{4}\right\}\right),\left(\left(\mathrm{p}_{2}, \mathrm{e}_{2}, 1\right),\left\{\mathrm{u}_{2}, \mathrm{u}_{5}\right\}\right),\left(\left(\neg \mathrm{p}_{2}, \mathrm{e}_{2}, 1\right),\left\{\mathrm{u}_{1}\right\}\right)
\end{array}\right\}
$$

and the disagree-BSES $(\Gamma, \Lambda, \mathrm{K})_{0}$ over $\mathrm{U}$ is

$$
(\Gamma, \Lambda, K)_{0}=\left\{\begin{array}{c}
\left(\left(\mathrm{p}_{1}, \mathrm{e}_{1}, 0\right),\left\{\mathrm{u}_{1}, \mathrm{u}_{4}, \mathrm{u}_{5}\right\}\right),\left(\left(\neg \mathrm{p}_{1}, \mathrm{e}_{2}, 0\right),\left\{\mathrm{u}_{2}, \mathrm{u}_{3}, \mathrm{u}_{5}\right\}\right),\left(\left(\mathrm{p}_{2}, \mathrm{e}_{1}, 0\right),\left\{\mathrm{u}_{1}, \mathrm{u}_{4}\right\}\right), \\
\left(\left(\neg \mathrm{p}_{2}, \mathrm{e}_{1}, 0\right),\left\{\mathrm{u}_{2}, \mathrm{u}_{3}, \mathrm{u}_{5}\right\}\right),\left(\left(\mathrm{p}_{2}, \mathrm{e}_{2}, 0\right),\left\{\mathrm{u}_{1}, \mathrm{u}_{3}, \mathrm{u}_{4}\right\}\right),\left(\left(\neg \mathrm{p}_{2}, \mathrm{e}_{2}, 0\right),\left\{\mathrm{u}_{2}, \mathrm{u}_{3}, \mathrm{u}_{4}, \mathrm{u}_{5}\right\}\right)
\end{array}\right\} .
$$

Definition 3.6. The complement of a BSES $(\Gamma, \Lambda, K)$ is denoted by $(\Gamma, \Lambda, K)^{\hat{c}}$ and is defined by $(\Gamma, \Lambda, \mathrm{K})^{\hat{\mathrm{c}}}=\left(\Gamma^{\hat{\mathrm{c}}}, \Lambda^{\hat{\mathrm{c}}}, \mathrm{K}\right)$ where $\Gamma^{\hat{\mathrm{c}}}$ and $\Lambda^{\hat{\mathrm{c}}}$ are mappings given by $\Gamma^{\hat{\mathrm{c}}}(\mathrm{p}, \mathrm{e}, 1)=\Gamma(\mathrm{p}, \mathrm{e}, 0)$ $\left(\Gamma^{\hat{\mathrm{c}}}(\mathrm{p}, \mathrm{e}, 0)=\Gamma(\mathrm{p}, \mathrm{e}, 1)\right)$ and $\Lambda^{\hat{\mathrm{c}}}(\neg \mathrm{p}, \mathrm{e}, 1)=\Lambda(\neg \mathrm{p}, \mathrm{e}, 0)\left(\Lambda^{\hat{\mathrm{c}}}(\neg \mathrm{p}, \mathrm{e}, 0)=\Lambda(\neg \mathrm{p}, \mathrm{e}, 1)\right), \forall \mathrm{p} \in \mathrm{P}, \forall \mathrm{e} \in \mathrm{E}$.

Proposition 3.1. If $(\Gamma, \Lambda, K)$ is a BSES over $U$, then

(1) $\left((\Gamma, \Lambda, K)^{\hat{c}}\right)^{\hat{c}}=(\Gamma, \Lambda, K)$,

(2) $(\Gamma, \Lambda, K)_{1}^{\hat{c}}=(\Gamma, \Lambda, K)_{0}$,

(3) $(\Gamma, \Lambda, K)_{0}^{\hat{c}}=(\Gamma, \Lambda, K)_{1}$.

Proof. The proof is straightforward.

Example 3.4. Consider the BSES $(\Gamma, \Lambda, Z)$ over $U$ given in Example 3.1. Then, we obtain 
$(\Gamma, \Lambda, Z)^{\hat{c}}=\left\{\begin{array}{c}\left(\left(\mathrm{p}_{1}, \mathrm{e}_{1}, 0\right),\left\{\mathrm{u}_{2}, \mathrm{u}_{3}\right\}\right),\left(\left(\neg \mathrm{p}_{1}, \mathrm{e}_{1}, 0\right),\left\{\mathrm{u}_{4}\right\}\right),\left(\left(\mathrm{p}_{1}, \mathrm{e}_{2}, 0\right),\left\{\mathrm{u}_{3}, \mathrm{u}_{5}\right\}\right), \\ \left(\left(\neg \mathrm{p}_{1}, \mathrm{e}_{2}, 0\right),\left\{\mathrm{u}_{1}, \mathrm{u}_{4}\right\}\right),\left(\left(\mathrm{p}_{2}, \mathrm{e}_{2}, 0\right),\left\{\mathrm{u}_{2}, \mathrm{u}_{5}\right\}\right),\left(\left(\neg \mathrm{p}_{2}, \mathrm{e}_{2}, 0\right),\left\{\mathrm{u}_{1}\right\}\right), \\ \left(\left(\mathrm{p}_{1}, \mathrm{e}_{1}, 1\right),\left\{\mathrm{u}_{1}, \mathrm{u}_{4}, \mathrm{u}_{5}\right\}\right),\left(\left(\neg \mathrm{p}_{1}, \mathrm{e}_{2}, 1\right),\left\{\mathrm{u}_{2}, \mathrm{u}_{3}, \mathrm{u}_{5}\right\}\right),\left(\left(\mathrm{p}_{2}, \mathrm{e}_{1}, 1\right),\left\{\mathrm{u}_{1}, \mathrm{u}_{4}\right\}\right), \\ \left(\left(\neg \mathrm{p}_{2}, \mathrm{e}_{1}, 1\right),\left\{\mathrm{u}_{2}, \mathrm{u}_{3}, \mathrm{u}_{5}\right\}\right),\left(\left(\mathrm{p}_{2}, \mathrm{e}_{2}, 1\right),\left\{\mathrm{u}_{1}, \mathrm{u}_{3}, \mathrm{u}_{4}\right\}\right),\left(\left(\neg \mathrm{p}_{2}, \mathrm{e}_{2}, 1\right),\left\{\mathrm{u}_{2}, \mathrm{u}_{3}, \mathrm{u}_{4}, \mathrm{u}_{5}\right\}\right)\end{array}\right\}$.

Definition 3.7. If $(\Gamma, \Lambda, K)$ and $\left(\Gamma_{1}, \Lambda_{1}, L\right)$ are two BSESs over $U$ then " $(\Gamma, \Lambda, K)$ AND $\left(\Gamma_{1}, \Lambda_{1}, L\right) "$ denoted $(\Gamma, \Lambda, K) \widehat{\Lambda}\left(\Gamma_{1}, \Lambda_{1}, \mathrm{~L}\right)$ is defined by

$(\Gamma, \Lambda, \mathrm{K}) \widehat{\Lambda}\left(\Gamma_{1}, \Lambda_{1}, \mathrm{~L}\right)=\left(\Gamma_{2}, \Lambda_{2}, \mathrm{~K} \times \mathrm{L}\right)$

where

and

$$
\Gamma_{2}\left(\left(p^{\mathrm{k}}, \mathrm{e}^{\mathrm{k}}, \mathrm{o}^{\mathrm{k}}\right),\left(\mathrm{p}^{\mathrm{l}}, \mathrm{e}^{\mathrm{l}}, \mathrm{o}^{\mathrm{l}}\right)\right)=\Gamma\left(\mathrm{p}^{\mathrm{k}}, \mathrm{e}^{\mathrm{k}}, \mathrm{o}^{\mathrm{k}}\right) \cap \Gamma_{1}\left(\mathrm{p}^{\mathrm{l}}, \mathrm{e}^{\mathrm{l}}, \mathrm{o}^{\mathrm{l}}\right)
$$

$$
\Lambda_{2}\left(\left(\neg \mathrm{p}^{\mathrm{k}}, \mathrm{e}^{\mathrm{k}}, \mathrm{o}^{\mathrm{k}}\right),\left(\neg \mathrm{p}^{\mathrm{l}}, \mathrm{e}^{\mathrm{l}}, \mathrm{o}^{\mathrm{l}}\right)\right)=\Lambda\left(\neg \mathrm{p}^{\mathrm{k}}, \mathrm{e}^{\mathrm{k}}, \mathrm{o}^{\mathrm{k}}\right) \cup \Lambda_{1}\left(\neg \mathrm{p}^{\mathrm{l}}, \mathrm{e}^{\mathrm{l}}, \mathrm{o}^{\mathrm{l}}\right)
$$

for all $\left(\left(\mathrm{p}^{\mathrm{k}}, \mathrm{e}^{\mathrm{k}}, \mathrm{o}^{\mathrm{k}}\right),\left(\mathrm{p}^{\mathrm{l}}, \mathrm{e}^{\mathrm{l}}, \mathrm{o}^{\mathrm{l}}\right)\right) \in \mathrm{K} \times \mathrm{L},\left(\left(\neg \mathrm{p}^{\mathrm{k}}, \mathrm{e}^{\mathrm{k}}, \mathrm{o}^{\mathrm{k}}\right),\left(\neg \mathrm{p}^{\mathrm{l}}, \mathrm{e}^{\mathrm{l}}, \mathrm{o}^{\mathrm{l}}\right)\right) \in(\neg \mathrm{K}) \times(\neg \mathrm{L})\left(\mathrm{p}^{\mathrm{k}}, \mathrm{p}^{\mathrm{l}} \in \mathrm{P}, \mathrm{e}^{\mathrm{k}} \in\right.$

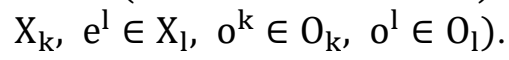

Definition 3.8. If $(\Gamma, \Lambda, K)$ and $\left(\Gamma_{1}, \Lambda_{1}, \mathrm{~L}\right)$ are two BSESs over $U$ then " $(\Gamma, \Lambda, K)$ OR $\left(\Gamma_{1}, \Lambda_{1}, \mathrm{~L}\right)$ " denoted $(\Gamma, \Lambda, \mathrm{K}) \widehat{\mathrm{V}}\left(\Gamma_{1}, \Lambda_{1}, \mathrm{~L}\right)$ is defined by

$(\Gamma, \Lambda, K) \widehat{V}\left(\Gamma_{1}, \Lambda_{1}, L\right)=\left(\Gamma_{2}, \Lambda_{2}, K \times L\right)$

where

and

$$
\Gamma_{2}\left(\left(p^{\mathrm{k}}, \mathrm{e}^{\mathrm{k}}, \mathrm{o}^{\mathrm{k}}\right),\left(\mathrm{p}^{\mathrm{l}}, \mathrm{e}^{\mathrm{l}}, \mathrm{o}^{\mathrm{l}}\right)\right)=\Gamma\left(\mathrm{p}^{\mathrm{k}}, \mathrm{e}^{\mathrm{k}}, \mathrm{o}^{\mathrm{k}}\right) \cup \Gamma_{1}\left(\mathrm{p}^{\mathrm{l}}, \mathrm{e}^{\mathrm{l}}, \mathrm{o}^{\mathrm{l}}\right)
$$

$$
\Lambda_{2}\left(\left(\neg \mathrm{p}^{\mathrm{k}}, \mathrm{e}^{\mathrm{k}}, \mathrm{o}^{\mathrm{k}}\right),\left(\neg \mathrm{p}^{\mathrm{l}}, \mathrm{e}^{\mathrm{l}}, \mathrm{o}^{\mathrm{l}}\right)\right)=\Lambda\left(\neg \mathrm{p}^{\mathrm{k}}, \mathrm{e}^{\mathrm{k}}, \mathrm{o}^{\mathrm{k}}\right) \cap \Lambda_{1}\left(\neg \mathrm{p}^{\mathrm{l}}, \mathrm{e}^{\mathrm{l}}, \mathrm{o}^{\mathrm{l}}\right)
$$

for all $\left(\left(\mathrm{p}^{\mathrm{k}}, \mathrm{e}^{\mathrm{k}}, \mathrm{o}^{\mathrm{k}}\right),\left(\mathrm{p}^{\mathrm{l}}, \mathrm{e}^{\mathrm{l}}, \mathrm{o}^{\mathrm{l}}\right)\right) \in \mathrm{K} \times \mathrm{L},\left(\left(\neg \mathrm{p}^{\mathrm{k}}, \mathrm{e}^{\mathrm{k}}, \mathrm{o}^{\mathrm{k}}\right),\left(\neg \mathrm{p}^{\mathrm{l}}, \mathrm{e}^{\mathrm{l}}, \mathrm{o}^{\mathrm{l}}\right)\right) \in(\neg \mathrm{K}) \times(\neg \mathrm{L})\left(\mathrm{p}^{\mathrm{k}}, \mathrm{p}^{\mathrm{l}} \in \mathrm{P}, \mathrm{e}^{\mathrm{k}} \in\right.$

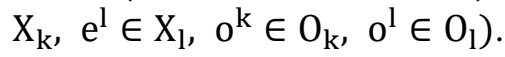

Proposition 3.2. If $(\Gamma, \Lambda, K)$ and $\left(\Gamma_{1}, \Lambda_{1}, \mathrm{~L}\right)$ are two BSESs over $\mathrm{U}$ then

(4) $\left((\Gamma, \Lambda, K) \widehat{\Lambda}\left(\Gamma_{1}, \Lambda_{1}, \mathrm{~L}\right)\right)^{\hat{\mathrm{c}}}=(\Gamma, \Lambda, \mathrm{K})^{\hat{\mathrm{c}}} \widehat{\mathrm{V}}\left(\Gamma_{1}, \Lambda_{1}, \mathrm{~L}\right)^{\hat{\mathrm{c}}}$,

(5) $\left((\Gamma, \Lambda, K) \widehat{V}\left(\Gamma_{1}, \Lambda_{1}, \mathrm{~L}\right)\right)^{\hat{\mathrm{c}}}=(\Gamma, \Lambda, \mathrm{K})^{\hat{\mathrm{c}}} \widehat{\Lambda}\left(\Gamma_{1}, \Lambda_{1}, \mathrm{~L}\right)^{\hat{\mathrm{c}}}$.

\section{Proof.}

(1) Suppose that $(\Gamma, \Lambda, K) \widehat{\Lambda}\left(\Gamma_{1}, \Lambda_{1}, \mathrm{~L}\right)=\left(\Gamma_{2}, \Lambda_{2}, \mathrm{~K} \times \mathrm{L}\right)$. Therefore, $\left(\Gamma_{2}, \Lambda_{2}, \mathrm{~K} \times \mathrm{L}\right)^{\hat{\mathrm{c}}}=\left(\Gamma_{2}^{\hat{c}}, \Lambda_{2}^{\hat{c}}, \mathrm{~K} \times \mathrm{L}\right)$, i.e.,

and

$$
\Gamma_{2}^{\hat{c}}\left(\left(\mathrm{p}^{\mathrm{k}}, \mathrm{e}^{\mathrm{k}}, \mathrm{o}^{\mathrm{k}}\right),\left(\mathrm{p}^{\mathrm{l}}, \mathrm{e}^{\mathrm{l}}, \mathrm{o}^{\mathrm{l}}\right)\right)=\left(\Gamma\left(\mathrm{p}^{\mathrm{k}}, \mathrm{e}^{\mathrm{k}}, \mathrm{o}^{\mathrm{k}}\right) \cap \Gamma_{1}\left(\mathrm{p}^{\mathrm{l}}, \mathrm{e}^{\mathrm{l}}, \mathrm{o}^{\mathrm{l}}\right)\right)^{\hat{c}}=\Gamma^{\hat{\mathrm{c}}}\left(\mathrm{p}^{\mathrm{k}}, \mathrm{e}^{\mathrm{k}}, \mathrm{o}^{\mathrm{k}}\right) \cup \Gamma_{1}^{\hat{c}}\left(\mathrm{p}^{\mathrm{l}}, \mathrm{e}^{\mathrm{l}}, \mathrm{o}^{\mathrm{l}}\right)
$$

$$
\begin{gathered}
\Lambda_{2}^{\hat{c}}\left(\left(\neg \mathrm{p}^{\mathrm{k}}, \mathrm{e}^{\mathrm{k}}, \mathrm{o}^{\mathrm{k}}\right),\left(\neg \mathrm{p}^{\mathrm{l}}, \mathrm{e}^{\mathrm{l}}, \mathrm{o}^{\mathrm{l}}\right)\right)=\left(\Lambda\left(\neg \mathrm{p}^{\mathrm{k}}, \mathrm{e}^{\mathrm{k}}, \mathrm{o}^{\mathrm{k}}\right) \cup \Lambda_{1}\left(\neg \mathrm{p}^{\mathrm{l}}, \mathrm{e}^{\mathrm{l}}, \mathrm{o}^{\mathrm{l}}\right)\right)^{\hat{c}} \\
=\Lambda^{\hat{\mathrm{c}}}\left(\neg \mathrm{p}^{\mathrm{k}}, \mathrm{e}^{\mathrm{k}}, \mathrm{o}^{\mathrm{k}}\right) \cap \Lambda_{1}^{\hat{c}}\left(\neg \mathrm{p}^{\mathrm{l}}, \mathrm{e}^{\mathrm{l}}, \mathrm{o}^{\mathrm{l}}\right)
\end{gathered}
$$

for all $\left(\left(\mathrm{p}^{\mathrm{k}}, \mathrm{e}^{\mathrm{k}}, \mathrm{o}^{\mathrm{k}}\right),\left(\mathrm{p}^{\mathrm{l}}, \mathrm{e}^{\mathrm{l}}, \mathrm{o}^{\mathrm{l}}\right)\right) \in \mathrm{K} \times \mathrm{L},\left(\left(\neg \mathrm{p}^{\mathrm{k}}, \mathrm{e}^{\mathrm{k}}, \mathrm{o}^{\mathrm{k}}\right),\left(\neg \mathrm{p}^{\mathrm{l}}, \mathrm{e}^{\mathrm{l}}, \mathrm{o}^{\mathrm{l}}\right)\right) \in(\neg \mathrm{K}) \times(\neg \mathrm{L})\left(\mathrm{p}^{\mathrm{k}}, \mathrm{p}^{\mathrm{l}} \in \mathrm{P}, \mathrm{e}^{\mathrm{k}} \in\right.$ $\left.X_{k}, e^{l} \in X_{l}, o^{k} \in O_{k}, o^{l} \in O_{l}\right)$. Here, let $o^{k}=1$ and $o^{l}=1$. Then,

$$
\Gamma^{\hat{\mathrm{c}}}\left(\mathrm{p}^{\mathrm{k}}, \mathrm{e}^{\mathrm{k}}, 1\right) \cup \Gamma_{1}^{\hat{c}}\left(\mathrm{p}^{\mathrm{l}}, \mathrm{e}^{\mathrm{l}}, 1\right)=\Gamma\left(\mathrm{p}^{\mathrm{k}}, \mathrm{e}^{\mathrm{k}}, 0\right) \cup \Gamma_{1}\left(\mathrm{p}^{\mathrm{l}}, \mathrm{e}^{\mathrm{l}}, 0\right)
$$

and

$$
\Lambda^{\hat{\mathrm{c}}}\left(\neg \mathrm{p}^{\mathrm{k}}, \mathrm{e}^{\mathrm{k}}, 1\right) \cap \Lambda_{1}^{\hat{c}}\left(\neg \mathrm{p}^{\mathrm{l}}, \mathrm{e}^{\mathrm{l}}, 1\right)=\Lambda\left(\neg \mathrm{p}^{\mathrm{k}}, \mathrm{e}^{\mathrm{k}}, 0\right) \cap \Lambda_{1}\left(\neg \mathrm{p}^{\mathrm{l}}, \mathrm{e}^{\mathrm{l}}, 0\right)
$$

On the other hand, let $(\Gamma, \Lambda, \mathrm{K})^{\hat{\mathrm{c}}} \widehat{\mathrm{V}}\left(\Gamma_{1}, \Lambda_{1}, \mathrm{~L}\right)^{\hat{\mathrm{c}}}=\left(\Gamma^{\hat{\mathrm{c}}}, \Lambda^{\hat{\mathrm{c}}}, \mathrm{K}\right) \widehat{\mathrm{V}}\left(\Gamma_{1}^{\hat{c}}, \Lambda_{1}^{\hat{c}}, \mathrm{~L}\right)=\left(\Gamma_{3}, \Lambda_{3}, \mathrm{~K} \times \mathrm{L}\right)$, i.e., 


$$
\Gamma_{3}\left(\left(\mathrm{p}^{\mathrm{k}}, \mathrm{e}^{\mathrm{k}}, 1\right),\left(\mathrm{p}^{\mathrm{l}}, \mathrm{e}^{\mathrm{l}}, 1\right)\right)=\Gamma^{\hat{\mathrm{c}}}\left(\mathrm{p}^{\mathrm{k}}, \mathrm{e}^{\mathrm{k}}, 1\right) \cup \Gamma_{1}^{\hat{c}}\left(\mathrm{p}^{\mathrm{l}}, \mathrm{e}^{\mathrm{l}}, 1\right)=\Gamma\left(\mathrm{p}^{\mathrm{k}}, \mathrm{e}^{\mathrm{k}}, 0\right) \cup \Gamma_{1}\left(\mathrm{p}^{\mathrm{l}}, \mathrm{e}^{\mathrm{l}}, 0\right)
$$

and

$$
\Lambda_{3}\left(\left(\neg \mathrm{p}^{\mathrm{k}}, \mathrm{e}^{\mathrm{k}}, 1\right),\left(\neg \mathrm{p}^{\mathrm{l}}, \mathrm{e}^{\mathrm{l}}, 1\right)\right)=\Lambda^{\hat{\mathrm{c}}}\left(\neg \mathrm{p}^{\mathrm{k}}, \mathrm{e}^{\mathrm{k}}, 1\right) \cap \Lambda_{1}^{\hat{c}}\left(\neg \mathrm{p}^{\mathrm{l}}, \mathrm{e}^{\mathrm{l}}, 1\right)=\Lambda\left(\neg \mathrm{p}^{\mathrm{k}}, \mathrm{e}^{\mathrm{k}}, 0\right) \cap \Lambda_{1}\left(\neg \mathrm{p}^{\mathrm{l}}, \mathrm{e}^{\mathrm{l}}, 0\right)
$$

for all $\left(\left(\mathrm{p}^{\mathrm{k}}, \mathrm{e}^{\mathrm{k}}, \mathrm{o}^{\mathrm{k}}\right),\left(\mathrm{p}^{\mathrm{l}}, \mathrm{e}^{\mathrm{l}}, \mathrm{o}^{\mathrm{l}}\right)\right) \in \mathrm{K} \times \mathrm{L},\left(\left(\neg \mathrm{p}^{\mathrm{k}}, \mathrm{e}^{\mathrm{k}}, \mathrm{o}^{\mathrm{k}}\right),\left(\neg \mathrm{p}^{\mathrm{l}}, \mathrm{e}^{\mathrm{l}}, \mathrm{o}^{\mathrm{l}}\right)\right) \in(\neg \mathrm{K}) \times(\neg \mathrm{L})\left(\mathrm{p}^{\mathrm{k}}, \mathrm{p}^{\mathrm{l}} \in \mathrm{P}, \mathrm{e}^{\mathrm{k}} \in\right.$ $\left.X_{k}, e^{l} \in X_{l}, o^{k} \in O_{k}, o^{l} \in O_{l}\right)$. Similarly, it can be shown in the cases " $o^{k}=1$ and $o^{l}=0 "$ " " $o^{k}=0$ and $o^{1}=1 ", " o^{k}=0$ and $o^{l}=0 "$.

(2) It is similar to the proof of (1).

Definition 3.9. Extended union of two BSESs $(\Gamma, \Lambda, \mathrm{K})$ and $\left(\Gamma_{1}, \Lambda_{1}, \mathrm{~L}\right)$ over $\mathrm{U}$ is the BSES $\left(\Gamma_{2}, \Lambda_{2}, M\right)$ over $\mathrm{U}$, where $\mathrm{M}=\mathrm{K} \cup \mathrm{L}$ and $\forall \mathrm{p} \in \mathrm{P}, \mathrm{e} \in \mathrm{E}, \mathrm{o} \in \mathrm{O}$,

$\Gamma_{2}(p, e, o)= \begin{cases}\Gamma(p, e, o), & \text { if }(p, e, o) \in K-L \\ \Gamma_{1}(p, e, o), & \text { if }(p, e, o) \in L-K \\ \Gamma(p, e, o) \cup \Gamma_{1}(p, e, o), & \text { if }(p, e, o) \in K \cap L\end{cases}$

$\Lambda_{2}(\neg \mathrm{p}, \mathrm{e}, \mathrm{o})= \begin{cases}\Lambda(\neg \mathrm{p}, \mathrm{e}, \mathrm{o}), & \text { if }(\neg \mathrm{p}, \mathrm{e}, \mathrm{o}) \in \mathrm{K}-\mathrm{L} \\ \Lambda_{1}(\neg \mathrm{p}, \mathrm{e}, \mathrm{o}), & \text { if }(\neg \mathrm{p}, \mathrm{e}, \mathrm{o}) \in \mathrm{L}-\mathrm{K} . \\ \Lambda(\neg \mathrm{p}, \mathrm{e}, \mathrm{o}) \cap \Lambda_{1}(\neg \mathrm{p}, \mathrm{e}, \mathrm{o}), & \text { if }(\neg \mathrm{p}, \mathrm{e}, \mathrm{o}) \in \mathrm{K} \cap \mathrm{L}\end{cases}$

We denote it by $(\Gamma, \Lambda, K) \widehat{\sqcup}\left(\Gamma_{1}, \Lambda_{1}, \mathrm{~L}\right)=\left(\Gamma_{2}, \Lambda_{2}, \mathrm{M}\right)$.

Definition 3.10. Extended intersection of two BSESs $(\Gamma, \Lambda, K)$ and $\left(\Gamma_{1}, \Lambda_{1}, L\right)$ over $U$ is the BSES $\left(\Gamma_{2}, \Lambda_{2}, M\right)$ over $\mathrm{U}$, where $\mathrm{M}=\mathrm{K} \cup \mathrm{L}$ and $\forall \mathrm{p} \in \mathrm{P}, \mathrm{e} \in \mathrm{E}, \mathrm{o} \in \mathrm{O}$,

$\Gamma_{2}(p, e, o)= \begin{cases}\Gamma(p, e, o), & \text { if }(p, e, o) \in K-L \\ \Gamma_{1}(p, e, o), & \text { if }(p, e, o) \in L-K \\ \Gamma(p, e, o) \cap \Gamma_{1}(p, e, o), & \text { if }(p, e, o) \in K \cap L\end{cases}$

$\Lambda_{2}(\neg$ p, e, o $)= \begin{cases}\Lambda(\neg \mathrm{p}, \mathrm{e}, \mathrm{o}), & \text { if }(\neg \mathrm{p}, \mathrm{e}, \mathrm{o}) \in \mathrm{K}-\mathrm{L} \\ \Lambda_{1}(\neg \mathrm{p}, \mathrm{e}, \mathrm{o}), & \text { if }(\neg \mathrm{p}, \mathrm{e}, \mathrm{o}) \in \mathrm{L}-\mathrm{K} . \\ \Lambda(\neg \mathrm{p}, \mathrm{e}, \mathrm{o}) \cup \Lambda_{1}(\neg \mathrm{p}, \mathrm{e}, \mathrm{o}), & \text { if }(\neg \mathrm{p}, \mathrm{e}, \mathrm{o}) \in \mathrm{K} \cap \mathrm{L}\end{cases}$

We denote it by $(\Gamma, \Lambda, K) \widehat{\Pi}\left(\Gamma_{1}, \Lambda_{1}, \mathrm{~L}\right)=\left(\Gamma_{2}, \Lambda_{2}, \mathrm{M}\right)$.

Definition 3.11. Restricted union of two BSESs $(\Gamma, \Lambda, K)$ and $\left(\Gamma_{1}, \Lambda_{1}, L\right)$ over $U$ is the BSES $\left(\Gamma_{2}, \Lambda_{2}, \mathrm{M}\right)$ over $\mathrm{U}$, where $\mathrm{M}=\mathrm{K} \cap \mathrm{L}$ and $\forall \mathrm{p} \in \mathrm{P}, \mathrm{e} \in \mathrm{E}, \mathrm{o} \in \mathrm{O}$,

$\Gamma_{2}(p, e, o)=\Gamma(p, e, o) \cup \Gamma_{1}(p, e, o)$

and

$\Lambda_{2}(\neg \mathrm{p}, \mathrm{e}, \mathrm{o})=\Lambda(\neg \mathrm{p}, \mathrm{e}, \mathrm{o}) \cap \Lambda_{1}(\neg \mathrm{p}, \mathrm{e}, \mathrm{o})$

We denote it by $(\Gamma, \Lambda, K) \widehat{\sqcup}_{\Re}\left(\Gamma_{1}, \Lambda_{1}, L\right)=\left(\Gamma_{1}, \Lambda_{1}, M\right)$.

Definition 3.12. Restricted intersection of two BSESs $(\Gamma, \Lambda, K)$ and $\left(\Gamma_{1}, \Lambda_{1}, \mathrm{~L}\right)$ over $\mathrm{U}$ is the BSES $\left(\Gamma_{2}, \Lambda_{2}, \mathrm{M}\right)$ over $\mathrm{U}$, where $\mathrm{M}=\mathrm{K} \cap \mathrm{L}$ and $\forall \mathrm{p} \in \mathrm{P}, \mathrm{e} \in \mathrm{E}, \mathrm{o} \in \mathrm{O}$,

$\Gamma_{2}(p, e, o)=\Gamma(p, e, o) \cap \Gamma_{1}(p, e, o)$ 
and

$\Lambda_{2}(\neg \mathrm{p}, \mathrm{e}, \mathrm{o})=\Lambda(\neg \mathrm{p}, \mathrm{e}, \mathrm{o}) \cup \Lambda_{1}(\neg \mathrm{p}, \mathrm{e}, \mathrm{o})$.

We denote it by $(\Gamma, \Lambda, K) \widehat{\Pi}_{\mathfrak{R}}\left(\Gamma_{1}, \Lambda_{1}, L\right)=\left(\Gamma_{1}, \Lambda_{1}, M\right)$.

Proposition 3.3. If $\left(\Gamma_{1}, \Lambda_{1}, K\right),\left(\Gamma_{2}, \Lambda_{2}, L\right)$ and $\left(\Gamma_{3}, \Lambda_{3}, M\right)$ are three BSESs over $U$, then

(1) $\left(\Gamma_{1}, \Lambda_{1}, \mathrm{~K}\right) \star\left(\Gamma_{2}, \Lambda_{2}, \mathrm{~L}\right)=\left(\Gamma_{2}, \Lambda_{2}, \mathrm{~L}\right) \star\left(\Gamma_{1}, \Lambda_{1}, \mathrm{~K}\right)$,

(2) $\left(\Gamma_{1}, \Lambda_{1}, \mathrm{~K}\right) \star\left(\left(\Gamma_{2}, \Lambda_{2}, \mathrm{~L}\right) \star\left(\Gamma_{3}, \Lambda_{3}, \mathrm{M}\right)\right)=\left(\left(\Gamma_{1}, \Lambda_{1}, \mathrm{~K}\right) \star\left(\Gamma_{2}, \Lambda_{2}, \mathrm{~L}\right)\right) \star\left(\Gamma_{3}, \Lambda_{3}, \mathrm{M}\right)$

For all $\star \in\left\{\widehat{\mathrm{U}}, \widehat{\Pi}, \widehat{ப}_{\mathfrak{R}}, \widehat{\Pi}_{\mathfrak{R}}\right\}$.

Proof. The proof is straightforward.

Example 3.5. Let $\mathrm{U}=\left\{\mathrm{u}_{1}, \mathrm{u}_{2}, \mathrm{u}_{3}, \mathrm{u}_{4}, \mathrm{u}_{5}\right\}$ be the set of houses under consideration,

$$
\mathrm{P}=\left\{\mathrm{p}_{1} \text { : furnished, } \mathrm{p}_{2} \text { : in the green surroundings, } \mathrm{p}_{3} \text { : pleasant }\right\}
$$

be the set of parameters and $\mathrm{E}=\left\{\mathrm{e}_{1}, \mathrm{e}_{2}\right\}$ be a set of experts. Then

$$
\neg \mathrm{P}=\left\{\neg \mathrm{p}_{1} \text { : non furnished, } \neg \mathrm{p}_{2} \text { : not in the green surroundings, } \neg \mathrm{p}_{3} \text { : unpleasant }\right\} \text {. }
$$

Suppose that $K=\left\{\mathrm{p}_{1}, \mathrm{p}_{2}\right\}$ and $\mathrm{L}=\left\{\mathrm{p}_{2}, \mathrm{p}_{3}\right\}$. The BSESs $(\Gamma, \Lambda, K)$ and $\left(\Gamma_{1}, \Lambda_{1}, \mathrm{~L}\right)$ describe the "requirements of the houses" which Mr. A and Mrs. B are going to buy, respectively. Suppose that

$$
\begin{aligned}
& \Gamma\left(\mathrm{p}_{1}, \mathrm{e}_{1}, 1\right)=\left\{\mathrm{u}_{1}, \mathrm{u}_{5}\right\}, \quad \Gamma\left(\mathrm{p}_{2}, \mathrm{e}_{1}, 1\right)=\left\{\mathrm{u}_{1}, \mathrm{u}_{4}\right\}, \quad \Lambda\left(\neg \mathrm{p}_{1}, \mathrm{e}_{1}, 1\right)=\left\{\mathrm{u}_{2}, \mathrm{u}_{3}\right\}, \\
& \Lambda\left(\neg \mathrm{p}_{2}, \mathrm{e}_{1}, 1\right)=\left\{\mathrm{u}_{3}, \mathrm{u}_{5}\right\}, \quad \Gamma\left(\mathrm{p}_{1}, \mathrm{e}_{2}, 1\right)=\left\{\mathrm{u}_{1}, \mathrm{u}_{2}\right\}, \quad \Gamma\left(\mathrm{p}_{2}, \mathrm{e}_{2}, 1\right)=\left\{\mathrm{u}_{3}, \mathrm{u}_{4}\right\}, \\
& \Lambda\left(\neg \mathrm{p}_{1}, \mathrm{e}_{2}, 1\right)=\left\{\mathrm{u}_{4}\right\}, \quad \Lambda\left(\neg \mathrm{p}_{2}, \mathrm{e}_{2}, 1\right)=\left\{\mathrm{u}_{5}\right\}, \quad \Gamma\left(\mathrm{p}_{1}, \mathrm{e}_{1}, 0\right)=\left\{\mathrm{u}_{2}, \mathrm{u}_{3}, \mathrm{u}_{4}\right\}, \\
& \Gamma\left(\mathrm{p}_{2}, \mathrm{e}_{1}, 0\right)=\left\{\mathrm{u}_{2}, \mathrm{u}_{3}, \mathrm{u}_{5}\right\}, \quad \Lambda\left(\neg \mathrm{p}_{1}, \mathrm{e}_{1}, 0\right)=\left\{\mathrm{u}_{1}, \mathrm{u}_{4}, \mathrm{u}_{5}\right\}, \quad \Lambda\left(\neg \mathrm{p}_{2}, \mathrm{e}_{1}, 0\right)=\left\{\mathrm{u}_{1}, \mathrm{u}_{2}, \mathrm{u}_{4}\right\}, \\
& \Gamma\left(\mathrm{p}_{1}, \mathrm{e}_{2}, 0\right)=\left\{\mathrm{u}_{3}, \mathrm{u}_{4}, \mathrm{u}_{5}\right\}, \quad \Gamma\left(\mathrm{p}_{2}, \mathrm{e}_{2}, 0\right)=\left\{\mathrm{u}_{1}, \mathrm{u}_{2}, \mathrm{u}_{5}\right\}, \quad \Lambda\left(\neg \mathrm{p}_{1}, \mathrm{e}_{2}, 0\right)=\left\{\mathrm{u}_{1}, \mathrm{u}_{2}, \mathrm{u}_{3}, \mathrm{u}_{5}\right\}, \\
& \Lambda\left(\neg \mathrm{p}_{2}, \mathrm{e}_{2}, 0\right)=\left\{\mathrm{u}_{1}, \mathrm{u}_{2}, \mathrm{u}_{3}, \mathrm{u}_{4}\right\}
\end{aligned}
$$

and

$$
\begin{array}{lll}
\Gamma_{1}\left(\mathrm{p}_{2}, \mathrm{e}_{1}, 1\right)=\left\{\mathrm{u}_{1}, \mathrm{u}_{3}, \mathrm{u}_{4}\right\}, & \Gamma_{1}\left(\mathrm{p}_{3}, \mathrm{e}_{1}, 1\right)=\left\{\mathrm{u}_{3}, \mathrm{u}_{4}\right\}, \quad \Lambda_{1}\left(\neg \mathrm{p}_{2}, \mathrm{e}_{1}, 1\right)=\left\{\mathrm{u}_{5}\right\}, \\
\Lambda_{1}\left(\neg \mathrm{p}_{3}, \mathrm{e}_{1}, 1\right)=\left\{\mathrm{u}_{1}, \mathrm{u}_{5}\right\}, & \Gamma_{1}\left(\mathrm{p}_{2}, \mathrm{e}_{2}, 1\right)=\left\{\mathrm{u}_{3}\right\}, \quad \Gamma_{1}\left(\mathrm{p}_{3}, \mathrm{e}_{2}, 1\right)=\left\{\mathrm{u}_{4}, \mathrm{u}_{5}\right\}, \\
\Lambda_{1}\left(\neg \mathrm{p}_{2}, \mathrm{e}_{2}, 1\right)=\left\{\mathrm{u}_{2}, \mathrm{u}_{5}\right\}, & \Lambda_{1}\left(\neg \mathrm{p}_{3}, \mathrm{e}_{2}, 1\right)=\left\{\mathrm{u}_{3}\right\}, \quad \Gamma_{1}\left(\mathrm{p}_{2}, \mathrm{e}_{1}, 0\right)=\left\{\mathrm{u}_{2}, \mathrm{u}_{5}\right\}, \\
\Gamma_{1}\left(\mathrm{p}_{3}, \mathrm{e}_{1}, 0\right)=\left\{\mathrm{u}_{1}, \mathrm{u}_{2}, \mathrm{u}_{5}\right\}, & \Lambda_{1}\left(\neg \mathrm{p}_{2}, \mathrm{e}_{1}, 0\right)=\left\{\mathrm{u}_{1}, \mathrm{u}_{2}, \mathrm{u}_{3}, \mathrm{u}_{4}\right\}, \quad \Lambda_{1}\left(\neg \mathrm{p}_{3}, \mathrm{e}_{1}, 0\right)=\left\{\mathrm{u}_{2}, \mathrm{u}_{3}, \mathrm{u}_{4}\right\}, \\
\Gamma_{1}\left(\mathrm{p}_{2}, \mathrm{e}_{2}, 0\right)=\left\{\mathrm{u}_{1}, \mathrm{u}_{2}, \mathrm{u}_{4}, \mathrm{u}_{5}\right\}, \quad \Gamma_{1}\left(\mathrm{p}_{3}, \mathrm{e}_{2}, 0\right)=\left\{\mathrm{u}_{1}, \mathrm{u}_{2}, \mathrm{u}_{3}\right\}, \quad \Lambda_{1}\left(\neg \mathrm{p}_{2}, \mathrm{e}_{2}, 0\right)=\left\{\mathrm{u}_{1}, \mathrm{u}_{3}, \mathrm{u}_{4}\right\}, \\
\Lambda_{1}\left(\neg \mathrm{p}_{3}, \mathrm{e}_{2}, 0\right)=\left\{\mathrm{u}_{1}, \mathrm{u}_{2}, \mathrm{u}_{4}, \mathrm{u}_{5}\right\} . &
\end{array}
$$

Now, we apply operations which are mentioned above on BSESs $(\Gamma, \Lambda, K)$ and $\left(\Gamma_{1}, \Lambda_{1}, L\right)$. Let $(\Gamma, \Lambda, \mathrm{K}) \widehat{\sqcup}\left(\Gamma_{1}, \Lambda_{1}, \mathrm{~L}\right)=\left(\Gamma_{2}, \Lambda_{2}, \mathrm{~K} \cup \mathrm{L}\right)$. Then

$$
\begin{aligned}
& \Gamma_{2}\left(p_{1}, \mathrm{e}_{1}, 1\right)=\left\{\mathrm{u}_{1}, \mathrm{u}_{5}\right\}, \quad \Gamma_{2}\left(\mathrm{p}_{2}, \mathrm{e}_{1}, 1\right)=\left\{\mathrm{u}_{1}, \mathrm{u}_{3}, \mathrm{u}_{4}\right\}, \quad \Gamma_{2}\left(\mathrm{p}_{3}, \mathrm{e}_{1}, 1\right)=\left\{\mathrm{u}_{3}, \mathrm{u}_{4}\right\}, \\
& \Gamma_{2}\left(p_{1}, e_{2}, 1\right)=\left\{u_{1}, u_{2}\right\}, \quad \Gamma_{2}\left(p_{2}, e_{2}, 1\right)=\left\{u_{3}, u_{4}\right\}, \quad \Gamma_{2}\left(p_{3}, e_{2}, 1\right)=\left\{u_{4}, u_{5}\right\} \\
& \Gamma_{2}\left(\mathrm{p}_{1}, \mathrm{e}_{1}, 0\right)=\left\{\mathrm{u}_{2}, \mathrm{u}_{3}, \mathrm{u}_{4}\right\}, \quad \Gamma_{2}\left(\mathrm{p}_{2}, \mathrm{e}_{1}, 0\right)=\left\{\mathrm{u}_{2}, \mathrm{u}_{3}, \mathrm{u}_{5}\right\}, \quad \Gamma_{2}\left(\mathrm{p}_{3}, \mathrm{e}_{1}, 0\right)=\left\{\mathrm{u}_{1}, \mathrm{u}_{2}, \mathrm{u}_{5}\right\}, \\
& \Gamma_{2}\left(\mathrm{p}_{1}, \mathrm{e}_{2}, 0\right)=\left\{\mathrm{u}_{3}, \mathrm{u}_{4}, \mathrm{u}_{5}\right\}, \quad \Gamma_{2}\left(\mathrm{p}_{2}, \mathrm{e}_{2}, 0\right)=\left\{\mathrm{u}_{1}, \mathrm{u}_{2}, \mathrm{u}_{4}, \mathrm{u}_{5}\right\}, \quad \Gamma_{2}\left(\mathrm{p}_{3}, \mathrm{e}_{2}, 0\right)=\left\{\mathrm{u}_{1}, \mathrm{u}_{2}, \mathrm{u}_{3}\right\}
\end{aligned}
$$

and

$$
\begin{aligned}
& \Lambda_{2}\left(\neg \mathrm{p}_{1}, \mathrm{e}_{1}, 1\right)=\left\{\mathrm{u}_{2}, \mathrm{u}_{3}\right\}, \quad \Lambda_{2}\left(\neg \mathrm{p}_{2}, \mathrm{e}_{1}, 1\right)=\left\{\mathrm{u}_{3}, \mathrm{u}_{5}\right\}, \quad \Lambda_{2}\left(\neg \mathrm{p}_{3}, \mathrm{e}_{1}, 1\right)=\left\{\mathrm{u}_{1}, \mathrm{u}_{5}\right\}, \\
& \Lambda_{2}\left(\neg \mathrm{p}_{1}, \mathrm{e}_{2}, 1\right)=\left\{\mathrm{u}_{4}\right\}, \quad \Lambda_{2}\left(\neg \mathrm{p}_{2}, \mathrm{e}_{2}, 1\right)=\left\{\mathrm{u}_{2}, \mathrm{u}_{5}\right\}, \quad \Lambda_{2}\left(\neg \mathrm{p}_{3}, \mathrm{e}_{2}, 1\right)=\left\{\mathrm{u}_{3}\right\}, \\
& \Lambda_{2}\left(\neg \mathrm{p}_{1}, \mathrm{e}_{1}, 0\right)=\left\{\mathrm{u}_{1}, \mathrm{u}_{4}, \mathrm{u}_{5}\right\}, \quad \Lambda_{2}\left(\neg \mathrm{p}_{2}, \mathrm{e}_{1}, 0\right)=\left\{\mathrm{u}_{1}, \mathrm{u}_{2}, \mathrm{u}_{3}, \mathrm{u}_{4}\right\}, \quad \Lambda_{2}\left(\neg \mathrm{p}_{3}, \mathrm{e}_{1}, 0\right)=\left\{\mathrm{u}_{2}, \mathrm{u}_{3}, \mathrm{u}_{4}\right\},
\end{aligned}
$$


$\Lambda_{2}\left(\neg \mathrm{p}_{1}, \mathrm{e}_{2}, 0\right)=\left\{\mathrm{u}_{1}, \mathrm{u}_{2}, \mathrm{u}_{3}, \mathrm{u}_{5}\right\}, \Lambda_{2}\left(\neg \mathrm{p}_{2}, \mathrm{e}_{2}, 0\right)=\left\{\mathrm{u}_{1}, \mathrm{u}_{2}, \mathrm{u}_{3}, \mathrm{u}_{4}\right\}, \quad \Lambda_{2}\left(\neg \mathrm{p}_{3}, \mathrm{e}_{2}, 0\right)=\left\{\mathrm{u}_{1}, \mathrm{u}_{2}, \mathrm{u}_{4}, \mathrm{u}_{5}\right\}$.

Let $(\Gamma, \Lambda, \mathrm{K}) \widehat{\Pi}\left(\Gamma_{1}, \Lambda_{1}, \mathrm{~L}\right)=\left(\Gamma_{3}, \Lambda_{3}, \mathrm{~K} \cup \mathrm{L}\right)$. Then

$$
\begin{array}{ll}
\Gamma_{3}\left(\mathrm{p}_{1}, \mathrm{e}_{1}, 1\right)=\left\{\mathrm{u}_{1}, \mathrm{u}_{5}\right\}, & \Gamma_{3}\left(\mathrm{p}_{2}, \mathrm{e}_{1}, 1\right)=\left\{\mathrm{u}_{1}, \mathrm{u}_{4}\right\}, \quad \Gamma_{3}\left(\mathrm{p}_{3}, \mathrm{e}_{1}, 1\right)=\left\{\mathrm{u}_{3}, \mathrm{u}_{4}\right\}, \\
\Gamma_{3}\left(\mathrm{p}_{1}, \mathrm{e}_{2}, 1\right)=\left\{\mathrm{u}_{1}, \mathrm{u}_{2}\right\}, & \Gamma_{3}\left(\mathrm{p}_{2}, \mathrm{e}_{2}, 1\right)=\left\{\mathrm{u}_{3}\right\}, \quad \Gamma_{3}\left(\mathrm{p}_{3}, \mathrm{e}_{2}, 1\right)=\left\{\mathrm{u}_{4}, \mathrm{u}_{5}\right\}, \\
\Gamma_{3}\left(\mathrm{p}_{1}, \mathrm{e}_{1}, 0\right)=\left\{\mathrm{u}_{2}, \mathrm{u}_{3}, \mathrm{u}_{4}\right\}, \quad \Gamma_{3}\left(\mathrm{p}_{2}, \mathrm{e}_{1}, 0\right)=\left\{\mathrm{u}_{2}, \mathrm{u}_{5}\right\}, \quad \Gamma_{3}\left(\mathrm{p}_{3}, \mathrm{e}_{1}, 0\right)=\left\{\mathrm{u}_{1}, \mathrm{u}_{2}, \mathrm{u}_{5}\right\}, \\
\Gamma_{3}\left(\mathrm{p}_{1}, \mathrm{e}_{2}, 0\right)=\left\{\mathrm{u}_{3}, \mathrm{u}_{4}, \mathrm{u}_{5}\right\}, \quad \Gamma_{3}\left(\mathrm{p}_{2}, \mathrm{e}_{2}, 0\right)=\left\{\mathrm{u}_{1}, \mathrm{u}_{2}, \mathrm{u}_{5}\right\}, \quad \Gamma_{3}\left(\mathrm{p}_{3}, \mathrm{e}_{2}, 0\right)=\left\{\mathrm{u}_{1}, \mathrm{u}_{2}, \mathrm{u}_{3}\right\}
\end{array}
$$

and

$$
\begin{aligned}
& \Lambda_{3}\left(\neg \mathrm{p}_{1}, \mathrm{e}_{1}, 1\right)=\left\{\mathrm{u}_{2}, \mathrm{u}_{3}\right\}, \quad \Lambda_{3}\left(\neg \mathrm{p}_{2}, \mathrm{e}_{1}, 1\right)=\left\{\mathrm{u}_{5}\right\}, \quad \Lambda_{3}\left(\neg \mathrm{p}_{3}, \mathrm{e}_{1}, 1\right)=\left\{\mathrm{u}_{1}, \mathrm{u}_{5}\right\}, \\
& \Lambda_{3}\left(\neg \mathrm{p}_{1}, \mathrm{e}_{2}, 1\right)=\left\{\mathrm{u}_{4}\right\}, \quad \Lambda_{3}\left(\neg \mathrm{p}_{2}, \mathrm{e}_{2}, 1\right)=\left\{\mathrm{u}_{5}\right\}, \quad \Lambda_{3}\left(\neg \mathrm{p}_{3}, \mathrm{e}_{2}, 1\right)=\left\{\mathrm{u}_{3}\right\}, \\
& \Lambda_{3}\left(\neg \mathrm{p}_{1}, \mathrm{e}_{1}, 0\right)=\left\{\mathrm{u}_{1}, \mathrm{u}_{4}, \mathrm{u}_{5}\right\}, \quad \Lambda_{3}\left(\neg \mathrm{p}_{2}, \mathrm{e}_{1}, 0\right)=\left\{\mathrm{u}_{1}, \mathrm{u}_{2}, \mathrm{u}_{4}\right\}, \quad \Lambda_{3}\left(\neg \mathrm{p}_{3}, \mathrm{e}_{1}, 0\right)=\left\{\mathrm{u}_{2}, \mathrm{u}_{3}, \mathrm{u}_{4}\right\}, \\
& \Lambda_{3}\left(\neg \mathrm{p}_{1}, \mathrm{e}_{2}, 0\right)=\left\{\mathrm{u}_{1}, \mathrm{u}_{2}, \mathrm{u}_{3}, \mathrm{u}_{5}\right\}, \quad \Lambda_{3}\left(\neg \mathrm{p}_{2}, \mathrm{e}_{2}, 0\right)=\left\{\mathrm{u}_{1}, \mathrm{u}_{3}, \mathrm{u}_{4}\right\}, \\
& \Lambda_{3}\left(\neg \mathrm{p}_{3}, \mathrm{e}_{2}, 0\right)=\left\{\mathrm{u}_{1}, \mathrm{u}_{2}, \mathrm{u}_{4}, \mathrm{u}_{5}\right\} .
\end{aligned}
$$

Let $(\Gamma, \Lambda, \mathrm{K}) \widehat{\sqcup}_{\Re}\left(\Gamma_{1}, \Lambda_{1}, \mathrm{~L}\right)=\left(\Gamma_{4}, \Lambda_{4}, \mathrm{~K} \cap \mathrm{L}\right)$. Then

$$
\begin{array}{ll}
\Gamma_{4}\left(\mathrm{p}_{2}, \mathrm{e}_{1}, 1\right)=\left\{\mathrm{u}_{1}, \mathrm{u}_{3}, \mathrm{u}_{4}\right\}, & \Gamma_{4}\left(\mathrm{p}_{2}, \mathrm{e}_{2}, 1\right)=\left\{\mathrm{u}_{3}, \mathrm{u}_{4}\right\}, \\
\Gamma_{4}\left(\mathrm{p}_{2}, \mathrm{e}_{1}, 0\right)=\left\{\mathrm{u}_{2}, \mathrm{u}_{5}\right\}, & \Gamma_{4}\left(\mathrm{p}_{2}, \mathrm{e}_{2}, 0\right)=\left\{\mathrm{u}_{1}, \mathrm{u}_{2}, \mathrm{u}_{5}\right\}
\end{array}
$$

and

$$
\begin{aligned}
& \Lambda_{4}\left(\neg \mathrm{p}_{2}, \mathrm{e}_{1}, 1\right)=\left\{\mathrm{u}_{3}, \mathrm{u}_{5}\right\}, \quad \Lambda_{4}\left(\neg \mathrm{p}_{2}, \mathrm{e}_{2}, 1\right)=\left\{\mathrm{u}_{2}, \mathrm{u}_{5}\right\}, \\
& \Lambda_{4}\left(\neg \mathrm{p}_{2}, \mathrm{e}_{1}, 0\right)=\left\{\mathrm{u}_{1}, \mathrm{u}_{2}, \mathrm{u}_{4}\right\}, \quad \Lambda_{4}\left(\neg \mathrm{p}_{2}, \mathrm{e}_{2}, 0\right)=\left\{\mathrm{u}_{1}, \mathrm{u}_{3}, \mathrm{u}_{4}\right\} .
\end{aligned}
$$

Let $(\Gamma, \Lambda, K) \widehat{\Pi}_{\Re}\left(\Gamma_{1}, \Lambda_{1}, \mathrm{~L}\right)=\left(\Gamma_{5}, \Lambda_{5}, \mathrm{~K} \cap \mathrm{L}\right)$. Then

$$
\begin{aligned}
& \Gamma_{5}\left(\mathrm{p}_{2}, \mathrm{e}_{1}, 1\right)=\left\{\mathrm{u}_{1}, \mathrm{u}_{4}\right\}, \quad \Gamma_{5}\left(\mathrm{p}_{2}, \mathrm{e}_{2}, 1\right)=\left\{\mathrm{u}_{3}\right\} \\
& \Gamma_{5}\left(\mathrm{p}_{2}, \mathrm{e}_{1}, 0\right)=\left\{\mathrm{u}_{2}, \mathrm{u}_{3}, \mathrm{u}_{5}\right\}, \quad \Gamma_{5}\left(\mathrm{p}_{2}, \mathrm{e}_{2}, 0\right)=\left\{\mathrm{u}_{1}, \mathrm{u}_{2}, \mathrm{u}_{4}, \mathrm{u}_{5}\right\}
\end{aligned}
$$

and

$$
\begin{aligned}
& \Lambda_{5}\left(\neg \mathrm{p}_{2}, \mathrm{e}_{1}, 1\right)=\left\{\mathrm{u}_{5}\right\}, \quad \Lambda_{5}\left(\neg \mathrm{p}_{2}, \mathrm{e}_{2}, 1\right)=\left\{\mathrm{u}_{5}\right\}, \\
& \Lambda_{5}\left(\neg \mathrm{p}_{2}, \mathrm{e}_{1}, 0\right)=\left\{\mathrm{u}_{1}, \mathrm{u}_{2}, \mathrm{u}_{3}, \mathrm{u}_{4}\right\}, \quad \Lambda_{5}\left(\neg \mathrm{p}_{2}, \mathrm{e}_{2}, 0\right)=\left\{\mathrm{u}_{1}, \mathrm{u}_{2}, \mathrm{u}_{3}, \mathrm{u}_{4}\right\} .
\end{aligned}
$$

Let $(\Gamma, \Lambda, \mathrm{K}) \widehat{\mathrm{V}}\left(\Gamma_{1}, \Lambda_{1}, \mathrm{~L}\right)=\left(\Gamma_{6}, \Lambda_{6}, \mathrm{~K} \times \mathrm{L}\right)$. Then

$$
\begin{aligned}
& \Gamma_{6}\left(\left(p_{1}, \mathrm{e}_{1}, 1\right),\left(\mathrm{p}_{2}, \mathrm{e}_{1}, 1\right)\right)=\left\{\mathrm{u}_{1}, \mathrm{u}_{3}, \mathrm{u}_{4}, \mathrm{u}_{5}\right\}, \quad \Gamma_{6}\left(\left(\mathrm{p}_{1}, \mathrm{e}_{1}, 1\right),\left(\mathrm{e}_{3}, \mathrm{x}_{1}, 1\right)\right)=\left\{\mathrm{u}_{1}, \mathrm{u}_{3}, \mathrm{u}_{4}, \mathrm{u}_{5}\right\} \\
& \Gamma_{6}\left(\left(\mathrm{p}_{2}, \mathrm{e}_{1}, 1\right),\left(\mathrm{p}_{2}, \mathrm{e}_{1}, 1\right)\right)=\left\{\mathrm{u}_{1}, \mathrm{u}_{3}, \mathrm{u}_{4}\right\}, \quad \Gamma_{6}\left(\left(\mathrm{p}_{2}, \mathrm{e}_{1}, 1\right),\left(\mathrm{p}_{3}, \mathrm{e}_{1}, 1\right)\right)=\left\{\mathrm{u}_{1}, \mathrm{u}_{3}, \mathrm{u}_{4}\right\}, \\
& \Gamma_{6}\left(\left(\mathrm{p}_{1}, \mathrm{e}_{1}, 0\right),\left(\mathrm{p}_{2}, \mathrm{e}_{1}, 0\right)\right)=\mathrm{U}, \quad \Gamma_{6}\left(\left(\mathrm{p}_{1}, \mathrm{e}_{1}, 0\right),\left(\mathrm{p}_{3}, \mathrm{e}_{1}, 0\right)\right)=\mathrm{U}, \\
& \Gamma_{6}\left(\left(\mathrm{p}_{2}, \mathrm{e}_{1}, 0\right),\left(\mathrm{p}_{2}, \mathrm{e}_{1}, 0\right)\right)=\left\{\mathrm{u}_{2}, \mathrm{u}_{3}, \mathrm{u}_{5}\right\}, \quad \Gamma_{6}\left(\left(\mathrm{p}_{2}, \mathrm{e}_{1}, 0\right),\left(\mathrm{p}_{3}, \mathrm{e}_{1}, 0\right)\right)=\left\{\mathrm{u}_{1}, \mathrm{u}_{2}, \mathrm{u}_{3}, \mathrm{u}_{5}\right\}
\end{aligned}
$$

and

$$
\begin{aligned}
& \Lambda_{6}\left(\left(\neg \mathrm{p}_{1}, \mathrm{e}_{1}, 1\right),\left(\neg \mathrm{p}_{2}, \mathrm{e}_{1}, 1\right)\right)=\left\{\mathrm{u}_{2}, \mathrm{u}_{3}, \mathrm{u}_{5}\right\}, \quad \Lambda_{6}\left(\left(\neg \mathrm{p}_{1}, \mathrm{e}_{1}, 1\right),\left(\neg \mathrm{p}_{3}, \mathrm{e}_{1}, 1\right)\right)=\left\{\mathrm{u}_{1}, \mathrm{u}_{2}, \mathrm{u}_{3}, \mathrm{u}_{5}\right\}, \\
& \Lambda_{6}\left(\left(\neg \mathrm{p}_{2}, \mathrm{e}_{1}, 1\right),\left(\neg \mathrm{p}_{2}, \mathrm{e}_{1}, 1\right)\right)=\left\{\mathrm{u}_{3}, \mathrm{u}_{5}\right\}, \quad \Lambda_{6}\left(\left(\neg \mathrm{p}_{2}, \mathrm{e}_{1}, 1\right),\left(\neg \mathrm{p}_{3}, \mathrm{e}_{1}, 1\right)\right)=\left\{\mathrm{u}_{1}, \mathrm{u}_{3}, \mathrm{u}_{5}\right\}, \\
& \Lambda_{6}\left(\left(\neg \mathrm{p}_{1}, \mathrm{e}_{1}, 0\right),\left(\neg \mathrm{p}_{2}, \mathrm{e}_{1}, 0\right)\right)=\mathrm{U}, \quad \Lambda_{6}\left(\left(\neg \mathrm{p}_{1}, \mathrm{e}_{1}, 0\right),\left(\neg \mathrm{p}_{3}, \mathrm{e}_{1}, 0\right)\right)=\mathrm{U}, \\
& \Lambda_{6}\left(\left(\neg \mathrm{p}_{2}, \mathrm{e}_{1}, 0\right),\left(\neg \mathrm{p}_{2}, \mathrm{e}_{1}, 0\right)\right)=\mathrm{U}, \quad \Lambda_{6}\left(\left(\neg \mathrm{p}_{2}, \mathrm{e}_{1}, 0\right),\left(\neg \mathrm{p}_{3}, \mathrm{e}_{1}, 0\right)\right)=\left\{\mathrm{u}_{1}, \mathrm{u}_{2}, \mathrm{u}_{3}, \mathrm{u}_{4}\right\}
\end{aligned}
$$

and so on. Let $(\Gamma, \Lambda, K) \widehat{\Lambda}\left(\Gamma_{1}, \Lambda_{1}, L\right)=\left(\Gamma_{7}, \Lambda_{7}, K \times L\right)$. Then 


$$
\begin{array}{ll}
\Gamma_{7}\left(\left(\mathrm{p}_{1}, \mathrm{e}_{1}, 1\right),\left(\mathrm{p}_{2}, \mathrm{e}_{1}, 1\right)\right)=\left\{\mathrm{u}_{1}\right\}, & \Gamma_{7}\left(\left(\mathrm{p}_{1}, \mathrm{e}_{1}, 1\right),\left(\mathrm{p}_{3}, \mathrm{e}_{1}, 1\right)\right)=\emptyset \\
\Gamma_{7}\left(\left(\mathrm{p}_{2}, \mathrm{e}_{1}, 1\right),\left(\mathrm{p}_{2}, \mathrm{e}_{1}, 1\right)\right)=\left\{\mathrm{u}_{1}, \mathrm{u}_{4}\right\}, & \Gamma_{7}\left(\left(\mathrm{p}_{2}, \mathrm{e}_{1}, 1\right),\left(\mathrm{p}_{3}, \mathrm{e}_{1}, 1\right)\right)=\left\{\mathrm{u}_{4}\right\} \\
\Gamma_{7}\left(\left(\mathrm{p}_{1}, \mathrm{e}_{1}, 0\right),\left(\mathrm{p}_{2}, \mathrm{e}_{1}, 0\right)\right)=\left\{\mathrm{u}_{2}, \mathrm{u}_{3}\right\}, & \Gamma_{7}\left(\left(\mathrm{p}_{1}, \mathrm{e}_{1}, 0\right),\left(\mathrm{p}_{3}, \mathrm{e}_{1}, 0\right)\right)=\left\{\mathrm{u}_{2}, \mathrm{u}_{3}, \mathrm{u}_{4}\right\} \\
\Gamma_{7}\left(\left(\mathrm{p}_{2}, \mathrm{e}_{1}, 0\right),\left(\mathrm{p}_{2}, \mathrm{e}_{1}, 0\right)\right)=\left\{\mathrm{u}_{2}, \mathrm{u}_{5}\right\}, & \Gamma_{7}\left(\left(\mathrm{p}_{2}, \mathrm{e}_{1}, 0\right),\left(\mathrm{p}_{3}, \mathrm{e}_{1}, 0\right)\right)=\left\{\mathrm{u}_{2}\right\}
\end{array}
$$

and

$$
\begin{aligned}
& \Lambda_{7}\left(\left(\neg \mathrm{p}_{1}, \mathrm{e}_{1}, 1\right),\left(\neg \mathrm{p}_{2}, \mathrm{e}_{1}, 1\right)\right)=\emptyset, \quad \Lambda_{7}\left(\left(\neg \mathrm{p}_{1}, \mathrm{e}_{1}, 1\right),\left(\neg \mathrm{p}_{3}, \mathrm{e}_{1}, 1\right)\right)=\emptyset, \\
& \Lambda_{7}\left(\left(\neg \mathrm{p}_{2}, \mathrm{e}_{1}, 1\right),\left(\neg \mathrm{p}_{2}, \mathrm{e}_{1}, 1\right)\right)=\left\{\mathrm{u}_{5}\right\}, \quad \Lambda_{7}\left(\left(\neg \mathrm{p}_{2}, \mathrm{e}_{1}, 1\right),\left(\neg \mathrm{p}_{3}, \mathrm{e}_{1}, 1\right)\right)=\left\{\mathrm{u}_{5}\right\}, \\
& \Lambda_{7}\left(\left(\neg \mathrm{p}_{1}, \mathrm{e}_{1}, 0\right),\left(\neg \mathrm{p}_{2}, \mathrm{e}_{1}, 0\right)\right)=\left\{\mathrm{u}_{1}, \mathrm{u}_{4}\right\}, \quad \Lambda_{7}\left(\left(\neg \mathrm{p}_{1}, \mathrm{e}_{1}, 0\right),\left(\neg \mathrm{p}_{3}, \mathrm{e}_{1}, 0\right)\right)=\left\{\mathrm{u}_{4}\right\}, \\
& \Lambda_{7}\left(\left(\neg \mathrm{p}_{2}, \mathrm{e}_{1}, 0\right),\left(\neg \mathrm{p}_{2}, \mathrm{e}_{1}, 0\right)\right)=\left\{\mathrm{u}_{1}, \mathrm{u}_{2}, \mathrm{u}_{4}\right\}, \quad \Lambda_{7}\left(\left(\neg \mathrm{p}_{2}, \mathrm{e}_{1}, 0\right),\left(\neg \mathrm{p}_{3}, \mathrm{e}_{1}, 0\right)\right)=\left\{\mathrm{u}_{2}, \mathrm{u}_{4}\right\}
\end{aligned}
$$

and so on.

\section{AN APLICATION OF BIPOLAR SOFT EXPERT SETS}

In this section, we give an application of BSES theory in a decision-making problem. The uncertainty problem determined for this is given as follows:

A private company wants to hire the best staff among the applicant candidates. Let $U=$ $\left\{\mathrm{u}_{1}, \mathrm{u}_{2}, \mathrm{u}_{3}, \mathrm{u}_{4}, \mathrm{u}_{5}\right\}$ be the set of candidates applying for recruitment and $\mathrm{P}=$ $\left\{p_{1}\right.$ : experience, $p_{2}$ : effective speaking $\}$ be the set of parameters that the company wants to be in the candidate to hire. Then,

$$
\neg \mathrm{P}=\left\{\neg \mathrm{p}_{1} \text { : inexperienced, } \neg \mathrm{p}_{2} \text { : ineffective speaking }\right\} .
$$

Let $\mathrm{E}=\left\{\mathrm{e}_{1}, \mathrm{e}_{2}\right\}$ be a set of experts (committee members). Let the BSES $(\Gamma, \Lambda, \mathrm{P})$ describes the "Personality Analysis of Candidates" as:

$$
(\Gamma, \Lambda, P)=\left\{\begin{array}{c}
\left(\left(\mathrm{p}_{1}, \mathrm{e}_{1}, 1\right),\left\{\mathrm{u}_{1}, \mathrm{u}_{5}\right\}\right),\left(\left(\neg \mathrm{p}_{1}, \mathrm{e}_{1}, 1\right),\left\{\mathrm{u}_{2}, \mathrm{u}_{3}\right\}\right),\left(\left(\mathrm{p}_{1}, \mathrm{e}_{2}, 1\right),\left\{\mathrm{u}_{2}, \mathrm{u}_{4}, \mathrm{u}_{5}\right\}\right), \\
\left(\left(\neg \mathrm{p}_{1}, \mathrm{e}_{2}, 1\right),\left\{\mathrm{u}_{1}, \mathrm{u}_{3}\right\}\right),\left(\left(\mathrm{p}_{2}, \mathrm{e}_{1}, 1\right),\left\{\mathrm{u}_{5}\right\}\right),\left(\left(\neg \mathrm{p}_{2}, \mathrm{e}_{1}, 1\right),\left\{\mathrm{u}_{2}, \mathrm{u}_{4}\right\}\right),\left(\left(\mathrm{p}_{2}, \mathrm{e}_{2}, 1\right),\left\{\mathrm{u}_{1}\right\}\right), \\
\left(\left(\neg \mathrm{p}_{2}, \mathrm{e}_{2}, 1\right),\left\{\mathrm{u}_{3}, \mathrm{u}_{4}\right\}\right),\left(\left(\mathrm{p}_{1}, \mathrm{e}_{1}, 0\right),\left\{\mathrm{u}_{2}, \mathrm{u}_{3}, \mathrm{u}_{4}\right\}\right),\left(\left(\neg \mathrm{p}_{1}, \mathrm{e}_{1}, 0\right),\left\{\mathrm{u}_{1}, \mathrm{u}_{4}, \mathrm{u}_{5}\right\}\right), \\
\left(\left(\mathrm{p}_{1}, \mathrm{e}_{2}, 0\right),\left\{\mathrm{u}_{1}, \mathrm{u}_{3}\right\}\right),\left(\left(\neg \mathrm{p}_{1}, \mathrm{e}_{2}, 0\right),\left\{\mathrm{u}_{2}, \mathrm{u}_{4}, \mathrm{u}_{5}\right\}\right),\left(\left(\mathrm{p}_{2}, \mathrm{e}_{1}, 0\right),\left\{\mathrm{u}_{1}, \mathrm{u}_{2}, \mathrm{u}_{3}, \mathrm{u}_{4}\right\}\right), \\
\left(\left(\neg \mathrm{p}_{2}, \mathrm{e}_{1}, 0\right),\left\{\mathrm{u}_{1}, \mathrm{u}_{3}, \mathrm{u}_{5}\right\}\right),\left(\left(\mathrm{p}_{2}, \mathrm{e}_{2}, 0\right),\left\{\mathrm{u}_{2}, \mathrm{u}_{3}, \mathrm{u}_{4}, \mathrm{u}_{5}\right\}\right),\left(\left(\neg \mathrm{p}_{2}, \mathrm{e}_{2}, 0\right),\left\{\mathrm{u}_{1}, \mathrm{u}_{2}, \mathrm{u}_{5}\right\}\right)
\end{array}\right\} .
$$

In Tables 1 and 2, we present the agree-BSES (for $\left(\mathrm{p}_{\mathrm{m}}, \mathrm{e}_{\mathrm{n}}\right)$ and $\left.\left(\neg \mathrm{p}_{\mathrm{m}}, \mathrm{e}_{\mathrm{n}}\right)\right)$ and disagree-BSES (for $\left(\mathrm{p}_{\mathrm{m}}, \mathrm{e}_{\mathrm{n}}\right)$ and $\left.\left(\neg \mathrm{p}_{\mathrm{m}}, \mathrm{e}_{\mathrm{n}}\right)\right)$, respectively, such that if $" \mathrm{u}_{\mathrm{i}} \in \Gamma(\mathrm{p}, \mathrm{e}, \mathrm{o})_{1}$ or $\mathrm{u}_{\mathrm{i}} \in \Lambda(\neg \mathrm{p}, \mathrm{e}, \mathrm{o})_{1}$ then $\mathrm{u}_{\mathrm{ij}}=1$ otherwise $u_{i j}=0$, and if " $u_{i} \in \Gamma(p, e, o)_{0}$ or $u_{i} \in \Lambda(\neg p, e, o)_{0}$ " then $u_{i j}=1$ otherwise $u_{i j}=0$ where $u_{i j}$ are the entries in Tables 1 and $2,\left(\mathrm{~m}, \mathrm{n} \in \mathbb{Z}^{+}\right)$.

The following algorithm may be followed by the company to fill the position.

\section{Algorithm}

(1) Input the BSES $(\Gamma, \Lambda, P)$,

(2) Find an agree-BSES and a diasagree-BSES,

(3) Find $A_{j}=\sum_{i} u_{i j}$ for agree-BSES (for $\left(p_{m}, e_{n}\right)$ ),

(4) Find $\mathrm{B}_{\mathrm{j}}=\sum_{\mathrm{i}} \mathrm{u}_{\mathrm{ij}}$ for agree-BSES (for $\left(\neg \mathrm{p}_{\mathrm{m}}, \mathrm{e}_{\mathrm{n}}\right)$ ),

(5) Find $\mathrm{C}_{\mathrm{j}}=\sum_{\mathrm{i}} \mathrm{u}_{\mathrm{ij}}$ for disagree-BSES (for $\left(\mathrm{p}_{\mathrm{m}}, \mathrm{e}_{\mathrm{n}}\right)$ ),

(6) Find $\mathrm{D}_{\mathrm{j}}=\sum_{\mathrm{i}} \mathrm{u}_{\mathrm{ij}}$ for disagree-BSES (for $\left(\neg \mathrm{p}_{\mathrm{m}}, \mathrm{e}_{\mathrm{n}}\right)$ ),

(7) Find $\left(A_{j}-B_{j}\right)-\left(C_{j}-D_{j}\right)=S_{j}$,

(8) Find $k$, for which $S_{k}=\max S_{j}$. 
Table 1. Agree-BSES (for $\left(p_{m}, e_{n}\right)$ and $\left.\left(\neg p_{m}, e_{n}\right)\right)$

\begin{tabular}{|l|c|r|r|r|r|l|r|r|r|r|r|}
\hline $\mathbf{U}$ & $\mathbf{u}_{\mathbf{1}}$ & $\mathbf{u}_{\mathbf{2}}$ & $\mathbf{u}_{\mathbf{3}}$ & $\mathbf{u}_{\mathbf{4}}$ & $\mathbf{u}_{\mathbf{5}}$ & $\mathbf{U}$ & $\mathbf{u}_{\mathbf{1}}$ & $\mathbf{u}_{\mathbf{2}}$ & $\mathbf{u}_{\mathbf{3}}$ & $\mathbf{u}_{\mathbf{4}}$ & $\mathbf{u}_{\mathbf{5}}$ \\
\hline$\left(\mathbf{p}_{\mathbf{1}}, \mathbf{e}_{\mathbf{1}}\right)$ & 1 & 0 & 0 & 0 & 1 & $\left(\neg \mathbf{p}_{\mathbf{1}}, \mathbf{e}_{\mathbf{1}}\right)$ & 0 & 1 & 1 & 0 & 0 \\
\hline$\left(\mathbf{p}_{\mathbf{1}}, \mathbf{e}_{2}\right)$ & 0 & 1 & 0 & 1 & 1 & $\left(\neg \mathbf{p}_{\mathbf{1}}, \mathbf{e}_{\mathbf{2}}\right)$ & 1 & 0 & 1 & 0 & 0 \\
\hline$\left(\mathbf{p}_{\mathbf{2}}, \mathbf{e}_{\mathbf{1}}\right)$ & 0 & 0 & 0 & 0 & 1 & $\left(\neg \mathbf{p}_{2}, \mathbf{e}_{\mathbf{1}}\right)$ & 0 & 1 & 0 & 1 & 0 \\
\hline$\left(\mathbf{p}_{\mathbf{2}}, \mathbf{e}_{\mathbf{2}}\right)$ & 1 & 0 & 0 & 0 & 0 & $\left(\neg \mathbf{p}_{\mathbf{2}}, \mathbf{e}_{\mathbf{2}}\right)$ & 0 & 0 & 1 & 1 & 0 \\
\hline $\mathbf{A}_{\mathbf{j}}=\sum_{\mathbf{i}} \mathbf{u}_{\mathbf{i j}}$ & 2 & 2 & 0 & 1 & 3 & $\mathbf{B}_{\mathbf{j}}=\sum_{\mathbf{i}} \mathbf{u}_{\mathbf{i j}}$ & 1 & 2 & 3 & 2 & 0 \\
\hline
\end{tabular}

Table 2. Disagree-BSES (for $\left(p_{m}, e_{n}\right)$ and $\left.\left(\neg p_{m}, e_{n}\right)\right)$

\begin{tabular}{|l|c|c|c|c|c|l|r|r|r|r|r|}
\hline $\mathbf{U}$ & $\mathbf{u}_{\mathbf{1}}$ & $\mathbf{u}_{\mathbf{2}}$ & $\mathbf{u}_{\mathbf{3}}$ & $\mathbf{u}_{\mathbf{4}}$ & $\mathbf{u}_{\mathbf{5}}$ & $\mathbf{U}$ & $\mathbf{u}_{\mathbf{1}}$ & $\mathbf{u}_{\mathbf{2}}$ & $\mathbf{u}_{\mathbf{3}}$ & $\mathbf{u}_{\mathbf{4}}$ & $\mathbf{u}_{\mathbf{5}}$ \\
\hline$\left(\mathbf{p}_{\mathbf{1}}, \mathbf{e}_{\mathbf{1}}\right)$ & 0 & 1 & 1 & 1 & 0 & $\left(\neg \mathbf{p}_{\mathbf{1}}, \mathbf{e}_{\mathbf{1}}\right)$ & 1 & 0 & 0 & 1 & 1 \\
\hline$\left(\mathbf{p}_{\mathbf{1}}, \mathbf{e}_{\mathbf{2}}\right)$ & 1 & 0 & 1 & 0 & 0 & $\left(\neg \mathbf{p}_{\mathbf{1}}, \mathbf{e}_{\mathbf{2}}\right)$ & 0 & 1 & 0 & 1 & 1 \\
\hline$\left(\mathbf{p}_{\mathbf{2}}, \mathbf{e}_{\mathbf{1}}\right)$ & 1 & 1 & 1 & 1 & 0 & $\left(\neg \mathbf{p}_{\mathbf{2}}, \mathbf{e}_{\mathbf{1}}\right)$ & 1 & 0 & 1 & 0 & 1 \\
\hline$\left(\mathbf{p}_{\mathbf{2}}, \mathbf{e}_{\mathbf{2}}\right)$ & 0 & 1 & 1 & 1 & 1 & $\left(\neg \mathbf{p}_{\mathbf{2}}, \mathbf{e}_{\mathbf{2}}\right)$ & 1 & 1 & 0 & 0 & 1 \\
\hline $\mathbf{C}_{\mathbf{j}}=\sum_{\mathbf{i}} \mathbf{u}_{\mathbf{i j}}$ & 2 & 3 & 4 & 3 & 1 & $\mathbf{D}_{\mathbf{j}}=\sum_{\mathbf{i}} \mathbf{u}_{\mathbf{i j}}$ & 3 & 2 & 1 & 2 & 4 \\
\hline
\end{tabular}

If the $\mathrm{k}$ value obtained here has more than one value, the company can choose any of them with their own option. Now let's use the proposed algorithm to find the candidate that best meets the parameters the private company wants, as given in Table 3 :

Table 3. Evaluation result

\begin{tabular}{|l|l|l|l|l|}
\hline $\mathbf{A}_{\mathbf{j}}$ & $\mathbf{B}_{\mathbf{j}}$ & $\mathbf{C}_{\mathbf{j}}$ & $\mathbf{D}_{\mathbf{j}}$ & $\mathbf{S}_{\mathbf{j}}$ \\
\hline $\mathrm{A}_{1}=2$ & $\mathrm{~B}_{1}=1$ & $\mathrm{C}_{1}=2$ & $\mathrm{D}_{1}=3$ & $\mathrm{~S}_{1}=2$ \\
\hline $\mathrm{A}_{2}=2$ & $\mathrm{~B}_{2}=2$ & $\mathrm{C}_{2}=3$ & $\mathrm{D}_{2}=2$ & $\mathrm{~S}_{2}=-1$ \\
\hline $\mathrm{A}_{3}=0$ & $\mathrm{~B}_{3}=3$ & $\mathrm{C}_{3}=4$ & $\mathrm{D}_{3}=1$ & $\mathrm{~S}_{3}=-6$ \\
\hline $\mathrm{A}_{4}=1$ & $\mathrm{~B}_{4}=2$ & $\mathrm{C}_{4}=3$ & $\mathrm{D}_{4}=2$ & $\mathrm{~S}_{4}=-2$ \\
\hline $\mathrm{A}_{5}=3$ & $\mathrm{~B}_{5}=0$ & $\mathrm{C}_{5}=1$ & $\mathrm{D}_{5}=4$ & $\mathrm{~S}_{5}=6$ \\
\hline
\end{tabular}

Here, it is recommended the private company hire the candidate $u_{5}$ for max $S_{k}=S_{5}$.

A comparison: Bipolar soft expert sets are more successful than soft expert sets in terms of a better expression of complex data that may be encountered in an uncertainty problem. Indeed, when we consider two decision making algorithms for soft expert sets available in the literature and our proposed approach in this paper to solve the above-mentioned problem, the ranking among objects is obtained as follows:

For Algorithm [15],

For Algorithm [17],

For the proposed algorithm,

$$
\begin{aligned}
& u_{1}=u_{2}=u_{3}<u_{4}<u_{5} \\
& u_{1}=u_{2}=u_{3}<u_{4}=u_{5}
\end{aligned}
$$

$$
u_{3}<u_{4}<u_{2}<u_{1}<u_{5} \text {. }
$$

Therefore, it is necessary to use the proposed algorithm in order to make the best separation between the objects. In this case, we emphasize that bipolar soft expert sets should be preferred in order to better express complex data.

\section{CONCLUSION}

In this paper, we introduce bipolar soft expert sets by using soft expert sets and bipolar soft sets. We also study basic operations such as subset, equal, complement, OR, AND, extended union, extended 
intersection, restricted union, restricted intersection. Thereafter, the basic properties of these operations are proven along with several examples to illustrate those properties. Finally, an algorithm based on bipolar soft expert sets was developed in order to better express a decision-making problem and an uncertainty problem was discussed to illustrate how this algorithm can be applied.

The researchers who will benefit from this paper in the future may be able to achieve more impressive results in applying different mathematical models to their decision-making problems regarding uncertainty situations. Thanks to the success of bipolar soft set and soft expert set especially in the area of uncertainty, we think that bipolar soft expert set we are working on will be an important research contribution.

\section{CONFLICTS OF INTEREST}

No conflict of interest was declared by the authors.

\section{REFERENCES}

[1] Zadeh, L. A., "Fuzzy sets”, Information \& Control, 8: 338-353, (1965).

[2] Pawlak, Z., "Rough sets", International Journal of Computing \& Information Sciences, 11: 341-356, (1982).

[3] Atanassov, K., “Intuitionistic fuzzy sets”, Fuzzy Sets \& System, 20: 87-96, (1986).

[4] Molodtsov, D., "Soft set theory first results", Computers and Mathematics with Applications, 37: 1931, (1999).

[5] Maji, P. K., Biswas, R., Roy, A. R., "Soft set theory", Computers and Mathematics with Applications, 45(4-5): 555-562, (2003).

[6] Demirtaş, N., Hussaın, S., Dalkılıç, O., "New approaches of inverse soft rough sets and their applications in a decision making problem", Journal of Applied Mathematics and Informatics, 38(34): 335-349, (2020).

[7] Demirtaş, N., Dalkılıç, O., "An application in the diagnosis of prostate cancer with the help of bipolar soft rough sets", on Mathematics and Mathematics Education (ICMME 2019), Konya, 283, (2019).

[8] Dalkılıç, O., Demirtas, N., "VFP-soft sets and its application on decision making problems", Journal of Polytechnic, (2021). DOI: https://doi.org/10.2339/politeknik.685634

[9] Dalkılıç, O., "An application of VFPFSS's in decision making problems", Journal of Polytechnic, (2021). DOI: https://doi.org/10.2339/politeknik.758474

[10] Dalk1lı̧, O., Demirtaş, N., "Bipolar soft filter", Journal of Universal Mathematics, 3(1): 21-27, (2020).

[11] Demirtaş, N., Dalkı1ıç, O., "Decompositions of soft $\alpha$-continuity and soft A-continuity", Journal of New Theory, (31): 86-94, (2020).

[12] Çağman, N., Enginoğlu, S., "Soft set theory and uni-int decision making", European Journal of Operational Research, 207: 848-855, (2010).

[13] Qin, K., Hong, Z., “On soft equality”, Journal of Computational and Applied Mathematics, 234: 1347$1355,(2010)$. 
[14] Majumdar, P., Samanta, S. K., "Similarity measure of soft sets", New Mathematics and Natural Computation, 4(1): 1-12, (2008).

[15] Alkhazaleh, S., Salleh, A. R., "Soft expert sets", Advances in Decision Sciences, Article ID757868, (2011).

[16] Alkhazaleh, S., Salleh, A. R., "Fuzzy soft expert set and its application", Applied Mathematics, 5: 1349-1368, (2014).

[17] Enginoğlu, S., Dönmez, H., “On soft expert sets”, Journal of New Theory, 9: 69-81, (2015).

[18] Çağman, N., Enginoğlu, S., "FP-soft set theory and its applications", Annals of Fuzzy Mathematics and informatics, 2(2): 219-226, (2011).

[19] Çağman, N., Enginoğlu, S., Çıtak, F., "Fuzzy soft set theory and its applications", Iranian Journal of Fuzzy Systems, 8(3): 137-147, (2011).

[20] Çağman, N., Karataş, S., "Intuitionistic fuzzy soft set theory and its decision making", Journal of Intelligent and Fuzzy Systems, 24(4): 829-836, (2013).

[21] Jiang, Y., Tang, Y., Chen, Q., Liu, H., Tang, J., "Interval-valued intuitionistic fuzzy soft sets and their properties", Computers and Mathematics with Applications, 60: 906-918, (2010).

[22] Jiang, Y., Tang, Y., Chen, Q., “An adjustable approach to intuitionistic fuzzy soft sets based decision making", Applied Mathematical Modelling, 35: 824-836, (2011).

[23] Maji, P. K., Biswas, R., Roy, A. R., "Fuzzy soft sets”, Journal of Fuzzy Mathematics, 9(3): 589-602, (2001).

[24] Yang, X., Lin, T. Y., Yang, J., Li, Y., Yu, D., "Combination of intervalvalued fuzzy set and soft set”, Computers and Mathematics with Applications, 58: 521-527, (2009).

[25] Kamac1, H. "Interval-valued fuzzy parameterized intuitionistic fuzzy soft sets and their applications", Cumhuriyet Science Journal, 40(2): 317-331, (2019).

[26] Ali, G., Akram, M., "Decision-making method based on fuzzy N-soft expert eets", Arabian Journal for Science and Engineering, 45(12): 10381-10400, (2020).

[27] Shabir, M., Naz, M., “On bipolar soft sets”, arXiv: 1303.1344v1 [math.LO], (2013).

[28] Karaaslan, F., Çağman, N., "Bipolar soft rough sets and their applications in decision making", Afrika Matematika, 29(5): 823-839, (2018).

[29] Karaaslan, F., "Bipolar soft rough relations", Communications Faculty of Sciences University of Ankara Series A1 Mathematics and Statistics, 65(1): 105-126, (2016).

[30] Karaaslan, F., Karataş, S., "A new approach to bipolar soft sets and its applications", Discrete Mathematics, Algorithms and Applications, 7(4): 1550054, (2015).

[31] Karaaslan, F., Ahmad, I., Ullah, A., "Bipolar soft groups", Journal of Intelligent and Fuzzy Systems, 31(1): 651-662, (2016). 